\title{
Optimizing the Accelerated Recursive Doubling Algorithm for Block Tridiagonal Systems of Equations
}

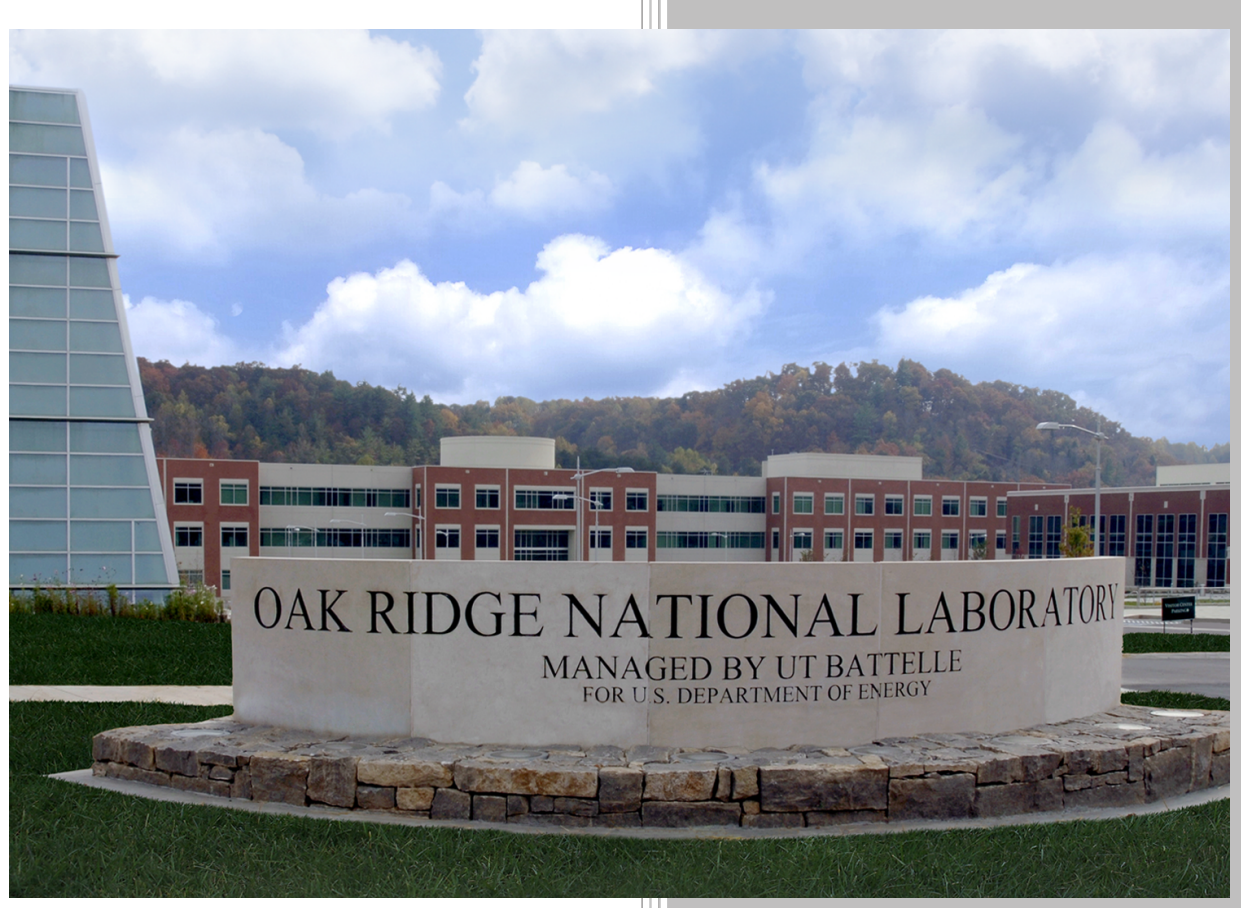

Approved for public release.

Muktaka Joshipura

Distribution is unlimited.

Sudip K Seal

August 7, 2020 


\section{DOCUMENT AVAILABILITY}

Reports produced after January 1, 1996, are generally available free via US Department of Energy (DOE) SciTech Connect.

Website: http://www.osti.gov/scitech/

Reports produced before January 1, 1996, may be purchased by members of the public from the following source:

National Technical Information Service

5285 Port Royal Road

Springfield, VA 22161

Telephone: 703-605-6000 (1-800-553-6847)

TDD: 703-487-4639

Fax: 703-605-6900

E-mail: info@ntis.gov

Website: http://classic.ntis.gov/

Reports are available to DOE employees, DOE contractors, Energy Technology Data Exchange representatives, and International Nuclear Information System representatives from the following source:

Office of Scientific and Technical Information

PO Box 62

Oak Ridge, TN 37831

Telephone: 865-576-8401

Fax: 865-576-5728

E-mail: report@osti.gov

Website: http://www.osti.gov/contact.html

This report was prepared as an account of work sponsored by an agency of the United States Government. Neither the United States Government nor any agency thereof, nor any of their employees, makes any warranty, express or implied, or assumes any legal liability or responsibility for the accuracy, completeness, or usefulness of any information, apparatus, product, or process disclosed, or represents that its use would not infringe privately owned rights. Reference herein to any specific commercial product, process, or service by trade name, trademark, manufacturer, or otherwise, does not necessarily constitute or imply its endorsement, recommendation, or favoring by the United States Government or any agency thereof. The views and opinions of authors expressed herein do not necessarily state or reflect those of the United States Government or any agency thereof. 


\title{
Computer Science and Mathematics Division
}

Optimizing the Accelerated Recursive Doubling Algorithm for Block Tridiagonal Systems of Equations

\author{
Muktaka Joshipura
}

Sudip K. Seal

Date Published: August, 2020

\author{
Prepared by \\ OAK RIDGE NATIONAL LABORATORY \\ Oak Ridge, TN 37831-6283 \\ managed by \\ UT-Battelle, LLC \\ for the \\ US DEPARTMENT OF ENERGY \\ under contract DE-AC05-00OR22725
}




\section{CONTENTS}

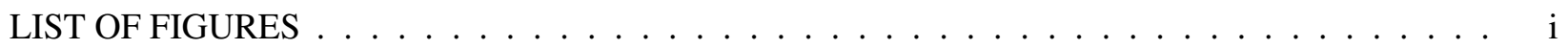

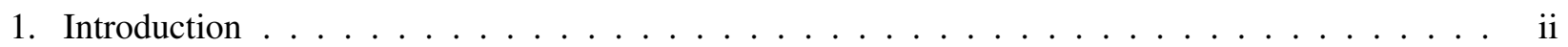

$1.1 \quad L_{i}, D_{i}$, and $U_{i}$ matrices $\ldots \ldots \ldots \ldots \ldots \ldots \ldots \ldots \ldots \ldots \ldots \ldots \ldots \ldots \ldots \ldots$

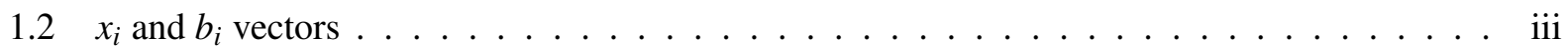

$1.3 X_{i}$ vectors and $B_{i}$ matrices $\ldots \ldots \ldots \ldots \ldots \ldots \ldots \ldots \ldots$ iii

$1.4 S_{i}$ matrices $\ldots \ldots \ldots \ldots \ldots \ldots \ldots \ldots \ldots \ldots$ iii

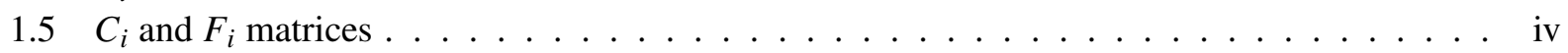

$1.6 Q_{i}$ matrices $\ldots \ldots \ldots \ldots \ldots \ldots \ldots \ldots \ldots \ldots \ldots \ldots \ldots$ iv

$1.7 E_{i}$ matrices $\ldots \ldots \ldots \ldots \ldots \ldots \ldots \ldots \ldots$

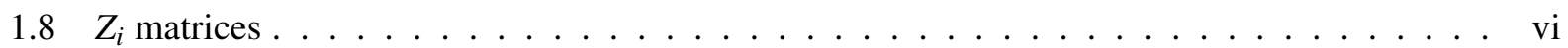

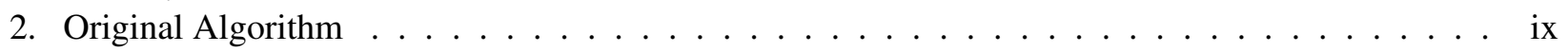

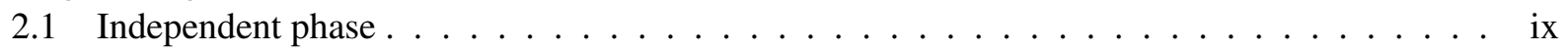

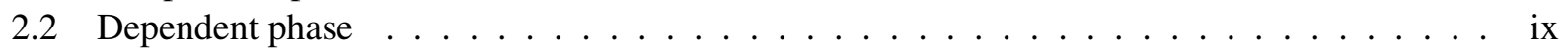

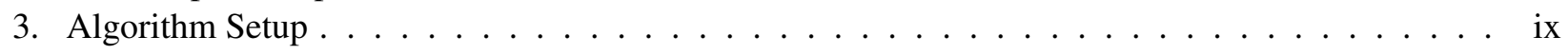

3.1 Block row assignment and indexing $\ldots \ldots \ldots \ldots \ldots \ldots \ldots$ ix

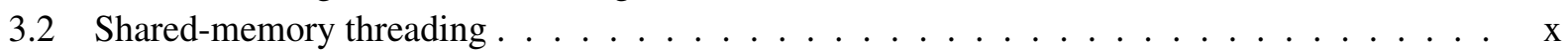

3.3 Registers and Instructions . . . . . . . . . . . . . . . . . . . . . . . .

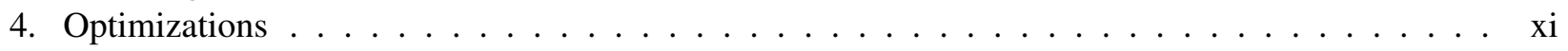

4.1 Optimizations in the Independent Phase $\ldots \ldots \ldots \ldots \ldots \ldots \ldots \ldots$

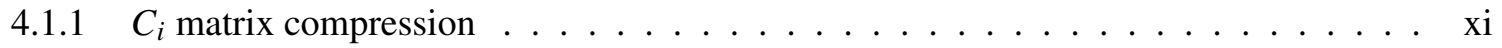

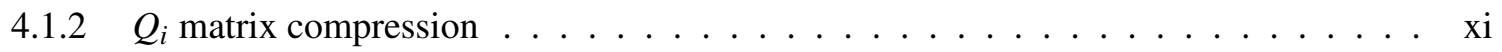

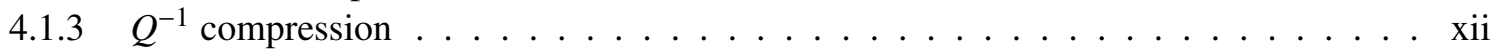

4.2 Optimization in the Dependent Phase . . . . . . . . . . . . . . xiii

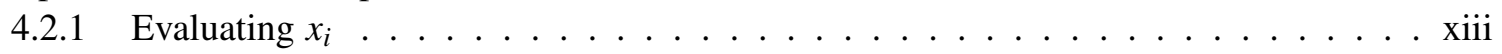

4.3 Shared-memory parallel prefix $\ldots \ldots \ldots \ldots \ldots \ldots \ldots$ xiv

4.3.1 Optimizations for in-place prefix operations . . . . . . . . . . . . xvi

4.3.2 Optimizations for out-of-place prefix operations . . . . . . . . . . . . xviii

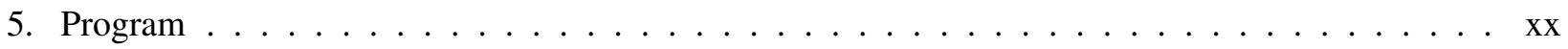

5.1 Independent Phase Part $1 \ldots \ldots \ldots \ldots \ldots \ldots \ldots \ldots$

5.2 Independent Phase Part $2 \ldots \ldots \ldots \ldots \ldots \ldots \ldots \ldots$ xxi

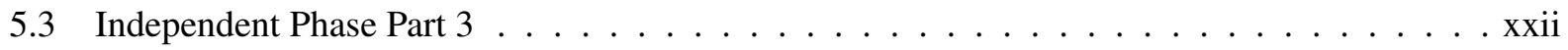

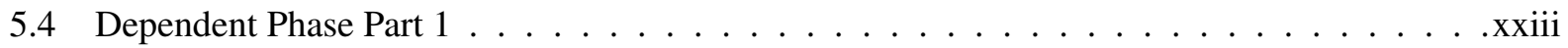

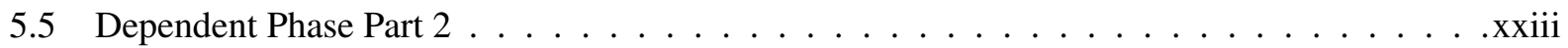

5.6 Dependent Phase Part $3 \ldots \ldots \ldots \ldots \ldots \ldots \ldots$. . . . . . . . . . . . . . . . . . . . . . . . . .

6. Time Complexity Analysis and Comparison . . . . . . . . . . . . . . . xxv

6.1 Naïve implementation . . . . . . . . . . . . . . . . . . . . xxv

6.1 .1 Independent phase $\ldots \ldots \ldots \ldots \ldots \ldots \ldots \ldots \ldots \ldots \ldots \ldots$

6.1 .2 Dependent Phase . . . . . . . . . . . . . . . . . . . . . . . . . . . . . . . . . . . .

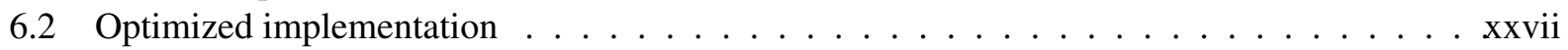

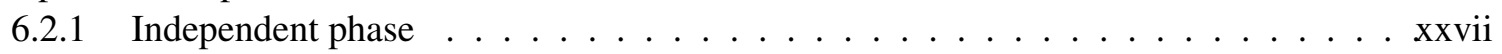

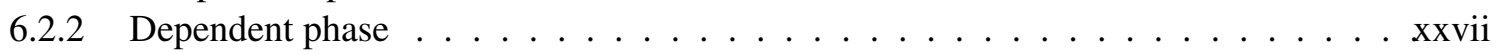

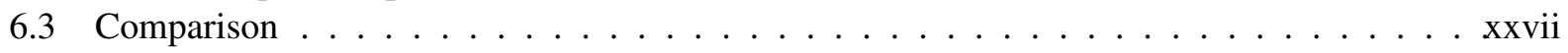

6.3 .1 Independent Phase . . . . . . . . . . . . . . . . . xxviii

6.3 .2 Dependent Phase . . . . . . . . . . . . . . . . . . xxviii 


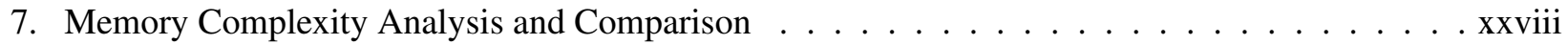

7.1 Naïve implementation . . . . . . . . . . . . . . . . . . xxviii

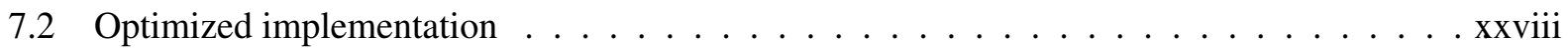

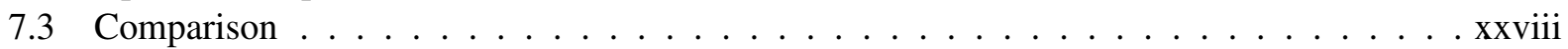

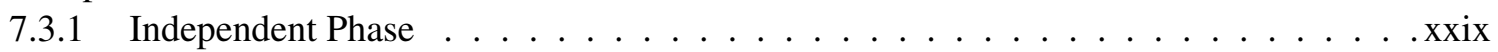

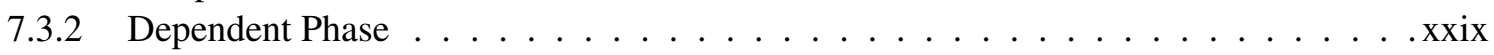

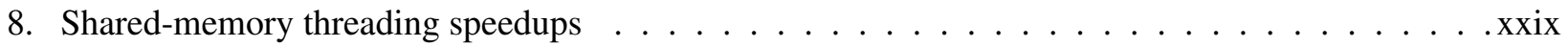

8.1 Independent phase . . . . . . . . . . . . . . . . . xxix

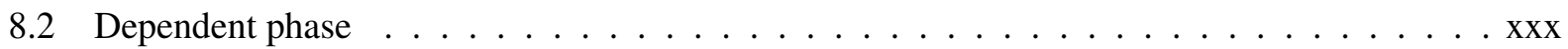

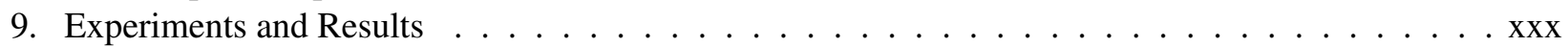

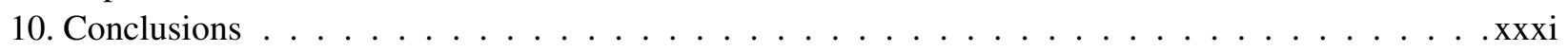




\section{LIST OF FIGURES}

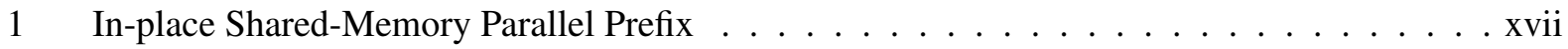

2 Out-of-place Shared-Memory Parallel Prefix . . . . . . . . . . . . . . . xix 


\begin{abstract}
The need to solve block tridiagonal systems with hundreds or thousands of right-hand sides for the same block tridiagonal matrix is common in a variety of disciplines. To meet this need, the Accelerated Recursive Doubling Algorithm was developed. After a right-hand side independent phase, the algorithm allows for the quick, online calculation of solutions for different right-hand sides. In this work, we present methods to optimize the Accelerated Recursive Doubling Algorithm in memory usage and computation time in a hybrid parallelization model. The right-hand side independent phase of the naïve implementation takes $\geq \frac{11}{3}$ the amount of memory required to store the tridiagonal matrix, while our implementation reduces the fraction to $\approx \frac{5}{3}$. The right-hand side dependent phase of the naïve implementation takes $\geq 6$ times the amount of memory required to store the right-hand side, while our implementation reduces the fraction to $\approx 3$. The computation time for the independent phase is reduced to $\approx \frac{2}{3}$ times that of the naïve implementation, while the computation time for the dependent phase is reduced to $\approx \frac{5}{9}$. With increasing numbers of shared-memory threads $q$ on every distributed processing element, we have $O(q)$ theoretical speedup.
\end{abstract}

\title{
1. Introduction
}

A block tridiagonal system is a linear system $A x=b$ such that $A$ is a block tridiagonal matrix. A block tridiagonal matrix is a block matrix such that the only non-zero blocks are on the block diagonal or adjacent to the main block diagonal.

To describe the dimensions of the matrix $A$, we define two parameters $M$ and $N . M$ refers to the width of each square block. $N$ refers to the number of block rows.

\section{$1.1 \quad L_{i}, D_{i}$, and $U_{i}$ matrices}

In order to reference specific blocks, we label each block in the block-tridiagonal matrix as either being 'lower' $(L)$, 'diagonal' $(D)$, or 'upper' $(U)$. We distinguish the blocks on each block row by a block-row index. Therefore, the layout of $A$ is as follows:

$$
A=\left[\begin{array}{ccccccc}
D_{1} & U_{1} & 0 & \cdots & \cdots & \cdots & 0 \\
L_{2} & D_{2} & U_{2} & 0 & \cdots & \cdots & 0 \\
0 & L_{3} & D_{3} & U_{3} & 0 & \cdots & 0 \\
\vdots & \ddots & \ddots & \ddots & \ddots & \ddots & \vdots \\
0 & \cdots & \cdots & \cdots & \cdots & L_{N} & D_{N}
\end{array}\right] .
$$

For convenience in using block-row indices, we define $L_{1}=U_{N}=I$. 


\section{$1.2 x_{i}$ and $b_{i}$ vectors}

We also section the solution vector $x$ and the right-hand side vector $b$ into smaller $M \times 1$ vectors, one for each of $N$ block rows, laid out as follows:

$$
x=\left[\begin{array}{c}
x_{1} \\
\vdots \\
x_{N}
\end{array}\right] \quad b=\left[\begin{array}{c}
b_{1} \\
\vdots \\
b_{N}
\end{array}\right] .
$$

Again, for convenience in using block-row indices, we define $x_{0}=x_{N+1}=0$.

\section{3 $X_{i}$ vectors and $B_{i}$ matrices}

Both the Accelerated and the non-accelerated Recursive Doubling Algorithms rely on a method of solving for $x_{i+1}$ once we have obtained a solution for $x_{i}$ and $x_{i-1}$, presented in [1]. This is done by multiplying a matrix $B_{i}$-determined from $L_{i}, D_{i}$ and $U_{i}$-with a vector $X_{i}$-determined from $x_{i}$ and $x_{i-1}$.

$X_{i}$ and $B_{i}$ were defined for $1 \leq i \leq N$ such that

$$
X_{i}=\left[\begin{array}{c}
x_{i} \\
x_{i-1} \\
1
\end{array}\right] \quad B_{i}=\left[\begin{array}{ccc}
-U_{i}^{-1} D_{i} & -U_{i}^{-1} L_{i} & U_{i}^{-1} b_{i} \\
I & 0 & 0 \\
0 & 0 & 1
\end{array}\right]
$$

We notice that the construction of $B_{i}$ requires the following assumption to be made:

Assumption 1. For all $1 \leq i \leq N$, the matrix $U_{i}$ is invertible.

The property that allows us to determine $x_{i+1}$ from $x_{i}$ and $x_{i-1}$ is stated as a lemma as

Lemma 1. For all $1 \leq i \leq N$, we have $X_{i+1}=B_{i} X_{i}$.

The proof of this lemma is found in [1].

This lemma tells us that once $x_{1}$ is known, we can pack $x_{1}$ and $x_{1-1}=x_{0}=0$ into $X_{1}$, calculate $X_{2}=B_{1} X_{1}$, and extract $x_{2}$ from the result. We can then calculate every subsequent $x_{i}$ in serial, thus obtaining the entirety of the solution vector $x$.

\section{$1.4 S_{i}$ matrices}

The property described in Lemma 1 yields the possibility of determining the value of all $x_{i}$ in parallel once $x_{1}$ is determined. This is used in both versions of the Recursive Doubling Algorithms. In order to do this, a prefix product of $B_{i}$, labeled $S_{i}$, is calculated, which is then multiplied with $X_{1}$ to obtain $X_{i}$. This property is presented in [1].

The prefix product $S_{i}=B_{i} B_{i-1} \ldots B_{1}$ is defined recursively for $1 \leq i \leq N$ as

$$
S_{i}=\left\{\begin{array}{ll}
B_{1} & i=1 \\
B_{i} S_{i-1} & i>1
\end{array} .\right.
$$

The property that allows $X_{i}$ to be calculated from $X_{1}$ using $S_{i}$ is stated as a theorem as 
Theorem 1. For all $1 \leq i \leq N$, we have $X_{i+1}=S_{i} X_{1}$.

This property follows trivially from the definition of $x_{i}$ in Equation 4 and the property of $B_{i}$ in Lemma 1 .

The method for determining $x_{1}$, and by extension $X_{1}$ as used in both versions of the Recursive Doubling Algorithm is

Theorem 2. $x_{1}=-\left[S_{N}^{11}\right]^{-1} S_{N}^{13}$ and so $X_{1}=\left[\begin{array}{c}-\left[S_{N}^{11}\right]^{-1} S_{N}^{13} \\ 0 \\ 1\end{array}\right]$.

A proof for this theorem can be found in [2].

However, the use of this property relies on the assumption that

Assumption 2. $S_{N}^{11}$ is invertible

\section{$1.5 C_{i}$ and $F_{i}$ matrices}

The Accelerated Recursive Doubling Algorithm presented in [2] accelerates the computation of solving for $x$ for multiple right-hand sides $b$ by separating the computation dependent on $b$ (right-hand side dependent) and the computation independent of the right-hand side. In order to do this, $B_{i}$ is separated into two matrices, one right-hand side independent $\left(C_{i}\right)$, and one right-hand side dependent $\left(F_{i}\right)$.

To be precise, for all $1 \leq i \leq N$, we decompose $B_{i}$ into $C_{i}$ and $F_{i}$ as

$$
C_{i}=\left[\begin{array}{ccc}
-U_{i}^{-1} D_{i} & -U_{i}^{-1} L_{i} & 0 \\
I & 0 & 0 \\
0 & 0 & 1
\end{array}\right] \quad F_{i}=\left[\begin{array}{ccc}
0 & 0 & U_{i}^{-1} b_{i} \\
0 & 0 & 0 \\
0 & 0 & 0
\end{array}\right]
$$

$C_{i}$ and $F_{i}$ matrices have the following properties that help simplify expressions involving their products, as presented in [2].

Lemma 2. For $1 \leq i \leq N$ and $1 \leq j \leq N$, we have $F_{i} F_{j}=0$.

Lemma 3. For $1 \leq i \leq N$ and $1 \leq j \leq N$, we have $F_{i} C_{j}=F_{i}$.

These lemmas are presented without proof because they can be verified trivially.

\section{6 $Q_{i}$ matrices}

In the Accelerated Recursive Doubling algorithm, $S_{i}$ is constructed by combining a result that only depends on $A$, and a result that only depends on the right-hand side $b$. The result that only depends on $A$ is the $Q_{i}$ matrix, which is the prefix product of $C_{i}$ matrices.

The prefix product $Q_{i}=C_{i} C_{i-1} \ldots C_{1}$ is defined recursively for $1 \leq i \leq N$ as

$$
Q_{i}= \begin{cases}C_{1} & i=1 \\ C_{i} Q_{i-1} & i>1\end{cases}
$$

$Q_{i}$ matrices have the following property that helps simplify expressions, stated as 
Lemma 4. For $1 \leq i \leq N$ and $1 \leq j \leq N$, we have $F_{i} Q_{j}=F_{i}$.

This lemma follows trivially from the definition of $Q_{i}$ and the property in Lemma 3.

\section{$1.7 \quad E_{i}$ matrices}

The right-hand side dependent result that is used to construct $S_{i}$ in the Accelerated Recursive Doubling Algorithm is the $E_{i}$ matrix, defined recursively for $1 \leq i \leq N$ as:

$$
E_{i}= \begin{cases}F_{1} & i=1 \\ F_{i}+C_{i} E_{i-1} & i>1\end{cases}
$$

Using this formulation, we can start from $E_{1}$ and determine all subsequent $E_{i}$ using the recursive formulation. This formulation of $E_{i}$ is simpler but equivalent to the original formulation in [2].

A property that helps simplify expressions is

Lemma 5. For $1 \leq i \leq N$ and $1 \leq j \leq N$, we have $F_{i} E_{j}=0$.

Proof. We can prove this property by induction.

Let $1 \leq i \leq N$ be a valid index.

Base case: $j=1$

By Lemma 2, we have

$$
F_{i} F_{1}=0
$$

\section{Inductive step}

Let the lemma be true for values of $j$ up to and including $k$. This means that $F_{i} E_{k}=0$. We have to prove that $F_{i} E_{k+1}=0$.

Since $k \geq 1$, we have $k+1 \geq 2$. So, by the definition of $E_{i}$, we have $E_{k+1}=F_{k+1}+C_{k+1} E_{k}$.

$$
\begin{aligned}
F_{i} E_{k+1} & =F_{i}\left(F_{k+1}+C_{k+1} E_{k}\right)=\left(F_{i} F_{k+1}\right)+\left(F_{i} C_{k+1}\right) E_{k} \\
& =0+F_{i} E_{k}(\text { By Lemma } 2 \text { and } 3) \\
& =0 \text { (By the inductive hypothesis }) .
\end{aligned}
$$

Using the value of $Q_{i}$, determined during the right-hand side independent computation, and the value of $E_{i}$, determined during the right-hand side dependent computation, we can construct the value of $S_{i}$ using this property.

Theorem 3. For all $1 \leq i \leq N$, we have $Q_{i}+E_{i}=S_{i}$.

Proof. We can prove this property using induction.

Base case: $i=1$ 
By definition of $S_{i}, Q_{i}$ and $E_{i}$, we have

$$
S_{1}=B_{1} \quad Q_{1}=C_{1} \quad E_{1}=F_{1}
$$

Therefore,

$$
Q_{1}+E_{1}=C_{1}+F_{1}=B_{1}=S_{1}
$$

\section{Inductive step}

Let the theorem be true for all $i$ up to and including $k$. Therefore, $S_{k}=Q_{k}+E_{k}$. We have to prove that $S_{k+1}=Q_{k+1}+E_{k+1}$.

By definition of $C_{i}$ and $F_{i}$, we have $Q_{k+1}=C_{k+1}+F_{k+1}$.

By definition of $S_{i}$ and the inductive hypothesis, we have

$$
S_{k+1}=B_{k+1} S_{k}=\left(C_{k+1}+F_{k+1}\right) S_{k}=\left(C_{k+1}+F_{k+1}\right)\left(Q_{k}+E_{k}\right)
$$

Expanding, we get

$$
\begin{aligned}
\left(C_{k+1}+F_{k+1}\right)\left(Q_{k}+E_{k}\right) & =C_{k+1} Q_{k}+C_{k+1} E_{k}+F_{k+1} Q_{k}+F_{k+1} E_{k} \\
& =Q_{k+1}+C_{k+1} E_{k}+F_{k+1}+0
\end{aligned}
$$

(Using Lemma 4 and Lemma 5)

Since $k \geq 1$, we know that $k+1>1$. So, we have $E_{k+1}=C_{k+1} E_{k}+F_{k+1}$. Plugging in the value of $E_{k+1}$, we get:

$$
S_{k+1}=Q_{k+1}+E_{k+1} \text {. }
$$

We have thus shown the inductive step to be true, which proves the theorem.

\section{8 $Z_{i}$ matrices}

The original formulation of $E_{i}$ allows for the calculation of all $E_{i}$ in parallel. For this formulation, we must define another kind of matrix, $Z_{i}$, defined for $1 \leq i \leq N$ as:

$$
Z_{i}=\sum_{k=1}^{i} Q_{i}^{-1} F_{i}
$$

Further, we define $Z_{0}=0$.

The existence of $Z_{i}$ therefore depends on the invertibility of $Q_{k}$ for all $1 \leq k \leq i$. In order to get the most minimal set of assumptions required to ensure this property, we investigated the conditions in which $Q_{i}$ are invertible. We discovered the following property.

Lemma 6. For $1 \leq i \leq N$, the matrix $Q_{i}$ is invertible if and only if $C_{1}, \ldots, C_{i}$ are invertible. 
Proof. By the properties of the determinant and the definition of $Q_{i}$ given in Equation 6, we have

$$
\operatorname{det} Q_{i}=\prod_{k=1}^{i} \operatorname{det} C_{i}
$$

Part 1: $C_{1}, \ldots, C_{i}$ are invertible $\Longrightarrow Q_{i}$ is invertible

If $C_{1}, \ldots, C_{i}$ are invertible, then $\operatorname{det} C_{1}, \ldots, \operatorname{det} C_{i}$ are non-zero. This means that the product of the determinants is also nonzero. Therefore, by Equation (9), we know that $\operatorname{det} Q_{i} \neq 0$, and so $Q_{i}$ is invertible.

Part 2: Not all of $C_{1}, \ldots, C_{i}$ are invertible $\Longrightarrow Q_{i}$ is not invertible

Let $C_{j} \in\left\{C_{1}, \ldots, C_{i}\right\}$ such that it is a non-invertible matrix. Then, $\operatorname{det} C_{j}=0$. By Equation (9), we know that $\operatorname{det} Q_{i}=0$, and so $Q_{i}$ is not invertible.

Since the invertibility of $Q_{i}$ matrices is conditional on the invertibility of $C_{i}$ matrices, we investigated the invertibility of $C_{i}$ matrices. We discovered the following property.

Lemma 7. Given that $U_{i}$ is invertible, for $1 \leq i \leq N$, the matrix $C_{i}$ is invertible if and only if $L_{i}$ is invertible.

Proof.

Part 1: $L_{i}$ is invertible $\Longrightarrow C_{i}$ is invertible

A matrix is invertible if and only its inverse exists. Let $L_{i}$ be invertible. Therefore $L^{-1}$ exists and so does the matrix

$$
D=\left[\begin{array}{ccc}
0 & I & 0 \\
-L_{i}^{-1} U_{i} & -L^{-1} D_{i} & 0 \\
0 & 0 & 1
\end{array}\right]
$$

Now, we have

$$
D C_{i}=\left[\begin{array}{ccc}
0 & I & 0 \\
-L_{i}^{-1} U_{i} & -L^{-1} D_{i} & 0 \\
0 & 0 & 1
\end{array}\right]\left[\begin{array}{ccc}
-U_{i}^{-1} D_{i} & -U_{i}^{-1} L_{i} & 0 \\
I & 0 & 0 \\
0 & 0 & 1
\end{array}\right]=I
$$

Therefore $D$ is the inverse of $C_{i}$ and $C_{i}$ is invertible.

Part 2: $L_{i}$ is not invertible $\Longrightarrow C_{i}$ is not invertible

A matrix $K$ is invertible if and only if there are no non-zero vectors $y$ such that $K y=0$. Since $L_{i}$ is not invertible, there is at least one non-zero vector $y$ such that $L_{i} y=0$.

Let $Y=\left[\begin{array}{l}0 \\ y \\ 0\end{array}\right]$.

Since $y \neq 0$, we know that $Y \neq 0$. 
Then,

$$
C_{i} Y=\left[\begin{array}{ccc}
-U_{i}^{-1} D_{i} & -U_{i}^{-1} L_{i} & 0 \\
I & 0 & 0 \\
0 & 0 & 1
\end{array}\right]\left[\begin{array}{l}
0 \\
y \\
0
\end{array}\right]=\left[\begin{array}{c}
-U_{i}^{-1} L_{i} y \\
0 \\
0
\end{array}\right]=\left[\begin{array}{l}
0 \\
0 \\
0
\end{array}\right]
$$

Since there exists a non-zero vector $Y$ such that $C_{i} Y=0$, we know that $C_{i}$ is not invertible.

Therefore, we were able to determine that the following condition is both necessary and sufficient for the invertibility of $Q_{i}$ and therefore the existence of $Z_{i}$ for all $1 \leq i \leq N$ :

Assumption 3. For $1 \leq i \leq N$, the matrix $L_{i}$ is invertible.

Note: A method suggested in [2] to make ARDA numerically stable for the same classes of matrices for which Cyclic Reduction is stable is to take the $L U$-decomposition of $A$, and then solve $A x=L U x=b$ in two steps: First solving $L y=b$, and then solve $U x=y$.

This is impossible to do by the ARDA algorithm since $L$ is lower-triangular, violating Assumption $1 U$ is upper-triangular, violating Assumption 3.

The original formulation of $E_{i}$ as described in [2] given in the theorem below, which also states the equivalence of the formulations described in this work and [2].

Theorem 4. For $1 \leq i \leq N$, the matrix $E_{i}=Q_{i} Z_{i-1}+F_{i}$.

Proof.

Base case: $i=1$

From Equation (7), we have

$$
E_{i}=E_{1}=F_{1} .
$$

The right hand side evaluates to

$$
Q_{i} Z_{i-1}+F_{i}=Q_{1} Z_{0}+F_{1}=0+F_{1}=F_{1} .
$$

Therefore, the proposition is true for $i=1$.

\section{Inductive step}

Assume that $E_{i}=Q_{i} Z_{i-1}+F_{i}$ for $i=k$. We have to prove that $E_{k+1}=Q_{k+1} Z_{k}+F_{k+1}$. From Equation (7), we have

$$
\begin{aligned}
E_{i+1} & =F_{i+1}+C_{i+1} E_{i}=F_{i+1}+C_{i+1}\left(Q_{i} Z_{i-1}+F_{i}\right) \\
& =F_{i+1}+C_{i+1}\left(Q_{i} Z_{i-1}+Q_{i} Q_{i}^{-1} F_{i}\right)=F_{i+1}+C_{i+1} Q_{i}\left(Z_{i-1}+Q_{i}^{-1} F_{i}\right) .
\end{aligned}
$$

By Equation (6), we have $C_{i+1} Q_{i}=Q_{i+1}$, and by Equation (8) we have $Z_{i-1}+Q_{i}^{-1} F_{i}=Z_{i}$. Therefore, we get

$$
E_{i+1}=Q_{i+1} Z_{i}+F_{i}
$$




\section{Original Algorithm}

The Accelerated Recursive Doubling Algorithm as described in [2] is described below for reference.

\subsection{Independent phase}

Algorithm 1: Independent Phase of the Non-optimized Accelerated Recursive Doubling Algorithm

1. Assign $K_{r}=\frac{N}{P}$ block rows of $A$ to each processor.

2. For each local block row $k$, compute $U_{k}^{-1}$, and use it to calculate $C_{k}$.

3. Calculate the local prefix product of the $C_{k}$ products to obtain local $Q_{k}$ 's.

4. Perform a prefix product of the total products across all processors.

5. Use the received prefix product to update the local prefixes to obtain global $Q_{k}$ 's.

6. Invert $Q_{k}$ to determine $Q_{k}^{-1}$.

\subsection{Dependent phase}

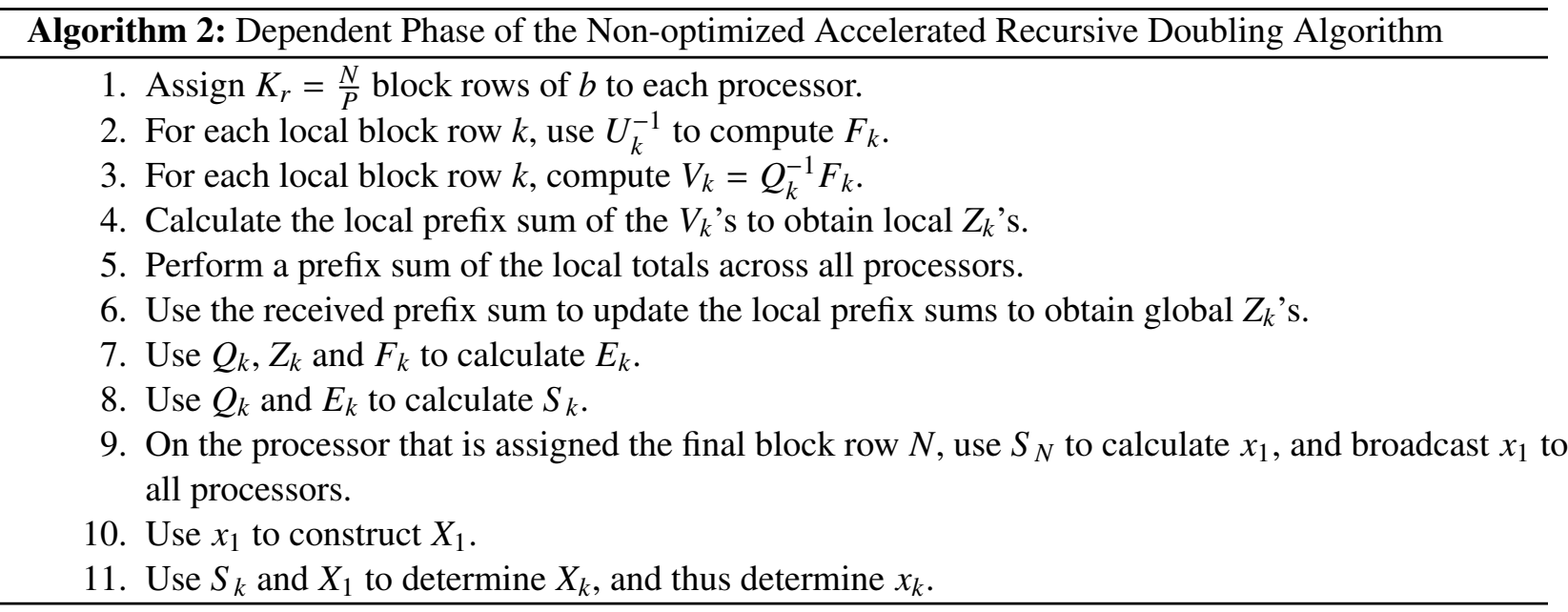

\section{Algorithm Setup}

\subsection{Block row assignment and indexing}

The $N$ block rows are distributed between the $P$ distributed processing elements evenly. The number of block rows assigned to a given processing element $r$ is $K_{r} \approx \frac{N}{P}$. Block rows can be indexed in two ways:

- Global index: Represented by $i$, this is the row index of the block row in $A$. The global index follows 1-based indexing.

- Local index: Represented by $k$, this is the index of the block row among the block rows assigned to the distributed processing element. The local index follows 0-based indexing. 


\subsection{Shared-memory threading}

The highly parallelizable nature of the Accelerated Recursive Doubling algorithm lends to the possibility of using shared-memory threads in conjunction with the distributed-memory algorithm described in [2].

We consider $q$ shared-memory threads running on every distributed processing element. The $K_{r}$ block rows assigned to the distributed processing element are sectioned evenly between the threads. The thread $t$ is responsible for block rows with local indexes $\mathrm{ts}_{t} \leq k<\mathrm{te}_{t}$.

\subsection{Registers and Instructions}

To increase the efficiency of the algorithm, the strategy chosen was to represent the algorithm in terms of atomic operations similar to those found in an assembly language. Operations like inverting, multiplying, and adding matrices become the 'instructions', while memory blocks of various sizes are considered to be 'registers'. Memory is optimized by reducing the number of 'registers' required, while computation is optimized by reducing the number or cost of the 'instructions' in the program.

In this implementation, memory is assigned once when the matrix $A$ is input, and freed once the solution to the right-hand side is output. All memory is contiguous, where consecutive registers have consecutive addresses in memory.

The memory is laid out with the following registers in order

1. Full block registers (size $M^{2} K_{r}$ each): $R^{0}, R^{1}, R^{2}, R^{3}, R^{4}$

- $R_{k}^{0}$ refers to the $k$-th block of size $M^{2}$ in $R^{0}$.

- $R^{01}$ refers to the register formed by combining registers $R^{0}$ and $R^{1}$ end-to-end.

- $R_{k}^{01}$ refers to the $k$-th block of size $2 M^{2}$ in $R^{01}$, interpreted as a matrix of dimensions $M \times 2 M$.

- $R_{k}^{01}[0]$ refers to the 0 -th block of size $M \times M$ in $R_{k}^{01}$.

- $R_{k}^{0}, R_{k}^{1}, R_{k}^{2}$ are assumed to be initialized with the appropriate $L_{i}, D_{i}$, and $U_{i}$ respectively, at the beginning of the independent phase execution.

2. Receive register $\left(\operatorname{size} 4 M^{2}\right): R^{r}$.

3. Thread registers (size $4 M^{2} q$ each): $R^{t 0}, R^{t 1}$

- $R_{t}^{t 0}$ refers to the $t$-th block of size $4 M^{2}$ in $R^{t 0}$.

- $R_{v 0}^{t 0}$ refers to the first block of size $2 M$ in $R^{t 0}$.

- $R_{v 1}^{t 0}$ refers to the block of size $M$ immediately after $R_{v 0}^{t 0}$.

4. Vector register (size $M K_{r}$ each): $R^{v 0}, R^{v 1}, R^{\nu 2}$.

- $R^{Z}$ refers to the register formed by combining registers $R^{v 0}$ and $R^{v 1}$ end-to-end.

- $R^{v 0}$ is assumed to be initialized with the appropriate $b_{i}$ at the beginning of every dependent phase execution. 


\section{Optimizations}

In this work, we present a large improvement in time and memory complexity of the algorithm. In order to do this, we exploit the structure and properties of the intermediate matrices and vectors calculated. We also present a method to efficiently perform parallel prefixes for the requirements of this algorithm in a shared-memory paradigm.

\subsection{Optimizations in the Independent Phase}

\subsection{1 $C_{i}$ matrix compression}

A $C_{i}$ matrix is determined from $L_{i}, D_{i}$, and $U_{i}$ matrices and is used as an input to a parallel prefix product algorithm in order to determine $Q_{i}$. $C_{i}$ matrices are of size $(2 M+1) \times(2 M+1)$, and are of the form

$$
C_{i}=\left[\begin{array}{ccc}
C_{i}^{11} & C_{i}^{12} & 0 \\
I & 0 & 0 \\
0 & 0 & 1
\end{array}\right]
$$

Therefore, $C_{i}$ can be compressed into a smaller matrix, given by

$$
C_{i}^{\prime}=\left[\begin{array}{ll}
C_{i}^{11} & C_{i}^{12}
\end{array}\right]
$$

A $C_{i}^{\prime}$ matrix can now be stored in a single cell of a concatenated register like $R^{01}$.

\subsection{2 $Q_{i}$ matrix compression}

A $Q$-type matrix is a matrix produced by the product of several $C_{i}$ matrices. They are of of size $(2 M+1) \times(2 M+1)$, and are of the form

$$
Q=\left[\begin{array}{ccc}
Q^{11} & Q^{12} & 0 \\
Q^{21} & Q^{22} & 0 \\
0 & 0 & 1
\end{array}\right]
$$

Therefore, a $Q_{i}$ matrix is a $Q$-type matrix.

These matrices are isomorphic over matrix multiplication to smaller $2 M \times 2 M$ matrices, which are constructed as

$$
Q^{\prime}=\left[\begin{array}{ll}
Q^{11} & Q^{12} \\
Q^{21} & Q^{22}
\end{array}\right]
$$

Theorem 5. If $Q_{A}$ is a $Q$-type matrix, and $C$ is a $C_{i}$ matrix, then $Q_{B}=C Q_{A}$ is such that the second block row of the $Q^{\prime}$ representation of $Q_{B}$ is equal to the first block row of the $Q^{\prime}$ representation of $Q_{A}$. 
Proof.

$$
Q_{B}=C Q_{A}
$$

Multiplying on the left on both sides of the above equation with $\left[\begin{array}{lll}0 & I & 0\end{array}\right]$, we get

$$
\begin{aligned}
{\left[\begin{array}{lll}
0 & I & 0
\end{array}\right]\left[\begin{array}{ccc}
Q_{B}^{11} & Q_{B}^{12} & 0 \\
Q_{B}^{21} & Q_{B}^{22} & 0 \\
0 & 0 & 1
\end{array}\right] } & =\left[\begin{array}{lll}
0 & I & 0
\end{array}\right]\left[\begin{array}{ccc}
C^{11} & C^{12} & 0 \\
I & 0 & 0 \\
0 & 0 & 1
\end{array}\right]\left[\begin{array}{ccc}
Q_{A}^{11} & Q_{A}^{12} & 0 \\
Q_{A}^{21} & Q_{A}^{22} & 0 \\
0 & 0 & 1
\end{array}\right] \\
{\left[\begin{array}{lll}
Q_{B}^{21} & Q_{B}^{22} & 0
\end{array}\right] } & =\left[\begin{array}{lll}
I & 0 & 0
\end{array}\right]\left[\begin{array}{ccc}
Q_{A}^{11} & Q_{A}^{12} & 0 \\
Q_{A}^{21} & Q_{A}^{22} & 0 \\
0 & 0 & 1
\end{array}\right] \\
{\left[\begin{array}{lll}
Q_{B}^{21} & Q_{B}^{22} & 0
\end{array}\right] } & =\left[\begin{array}{lll}
Q_{A}^{11} & Q_{A}^{12} & 0
\end{array}\right] .
\end{aligned}
$$

From the definition of $Q_{i}^{\prime}$ in Equation (13) and the above result, we get

$$
\begin{aligned}
Q_{B}^{\prime}=\left[\begin{array}{ll}
Q_{B}^{11} & Q_{B}^{12} \\
Q_{B}^{21} & Q_{B}^{22}
\end{array}\right] & =\left[\begin{array}{ll}
Q_{B}^{11} & Q_{B}^{12} \\
Q_{A}^{11} & Q_{A}^{12}
\end{array}\right] \\
Q_{i}^{\prime} & =\left[\begin{array}{ll}
Q_{A}^{11} & Q_{A}^{12} \\
Q_{A}^{21} & Q_{A}^{22}
\end{array}\right] .
\end{aligned}
$$

We observe that the second block row of $Q_{B}^{\prime}$ is the first block row of $Q_{A}^{\prime}$.

This means that if $Q_{1}^{\prime}, Q_{2}^{\prime}, \ldots, Q_{k}^{\prime}$ were the $Q^{\prime}$ representations of prefix product of some ordered collection of $C_{i}$ matrices, the second block row of $Q_{m}^{\prime}$ would be equal to the first block row of $Q_{m-1}^{\prime}$ if $i>1$, and would be equal to the first block row of the identity matrix $\left[\begin{array}{ll}I & 0\end{array}\right]$ if $i=0$.

This further means that when a $Q^{\prime}$ is calculated as a result of a prefix operation, and stored among adjacent prefixes, we can further compress the $Q^{\prime}$ matrix into a $Q^{\prime \prime}$ matrix given by

$$
Q^{\prime \prime}=\left[\begin{array}{ll}
Q^{11} & Q^{12}
\end{array}\right]
$$

In this case, since we only have to calculate the first block row, the time complexity of this multiplication is only $T_{C^{\prime} \times Q^{\prime}}=T_{Q^{\prime \prime} \times Q^{\prime}}=4 C_{\text {mul }} M^{3}$ instead of $8 C_{\text {mul }} M^{3}$.

However, when a $Q^{\prime}$ is calculated by multiplying two other $Q^{\prime}$ 's and not stored among adjacent prefixes, we need to calculate and store the full $Q^{\prime}$ result. The time complexity of this operation is therefore $T_{Q^{\prime} \times Q^{\prime}}=8 C_{\mathrm{mul}} M^{3}$.

For every block row $1 \leq i \leq N$, we define the $Q^{\prime \prime}$ representation of $Q_{i}$ as $Q_{i}^{\prime \prime}$.

\subsection{3 $Q^{-1}$ compression}

Because of the isomorphism, we know that even $Q_{i}^{-1}$ matrices are representable as $Q^{\prime}$ matrices, which are two block-rows wide. 
As described in [2], only the first block column of the inverse of a $Q_{i}^{-1}$ is used. Therefore, we can omit storing and calculating other block columns of the matrix.

Since the $Q^{\prime}$ representation of $Q_{i}$ (represented by $Q_{i}^{\prime}$ ) are block matrices, we can use the following property of block matrices

$$
Q_{i}^{\prime}=\left[\begin{array}{ll}
Q^{11} & Q^{12} \\
Q^{21} & Q^{22}
\end{array}\right]=\left[\begin{array}{ll}
A & B \\
C & D
\end{array}\right] \text { then } Q^{-1}=\left[\begin{array}{cc}
A^{-1}\left(I+B\left(D-C A^{-1} B\right)^{-1} C A^{-1}\right) & -A^{-1} B\left(D-C A^{-1} B\right) \\
-\left(D-C A^{-1} B\right)^{-1} C A^{-1} & \left(D-C A^{-1} B\right)^{-1}
\end{array}\right]
$$

Assuming that $A$ and $D-C A^{-1} B$ are invertible. This occurs when $Q_{i}^{11}$ and $Q_{i}^{22}-Q_{i}^{21}\left(Q_{i}^{11}\right)^{-1} Q_{i}^{12}$ are invertible.

We define $V=D-C A^{-1} B$ and $W=B V^{-1} C A^{-1}$ for brevity.

We therefore can calculate only the first block column of $Q_{i}^{-1}$ like so:

$$
Q_{i}^{-1}=\left[\begin{array}{c}
A^{-1}(I+W) \\
-V^{-1} C A^{-1}
\end{array}\right]
$$

However, this optimization requires the following assumption to be made:

Assumption 4. For $1 \leq i \leq N$,

\subsection{Optimization in the Dependent Phase}

\subsubsection{Evaluating $x_{i}$}

Theorem 6. For $1 \leq i \leq N$,

$$
x_{i}=Q_{i-1}^{\prime \prime}\left[\begin{array}{c}
x_{0}+Z_{i}^{13} \\
Z_{i}^{23}
\end{array}\right]
$$

Proof. From Theorem 1, we have

$$
\begin{aligned}
X_{i+1} & =S_{i} X_{i} \\
{\left[\begin{array}{c}
x_{i+1} \\
x_{i} \\
1
\end{array}\right] } & =\left(Q_{i}+E_{i}\right) X_{1}=\left(Q_{i}+Q_{i} Z_{i-1}+F_{i}\right) X_{1}=\left(Q_{i}\left(I+Z_{i-1}\right)+F_{i}\right) X_{1} \\
& =Q_{i}\left(I+Z_{i-1}\right) X_{1}+F_{i} X_{1} \\
& =\left[\begin{array}{ccc}
Q_{i}^{11} & Q_{i}^{12} & 0 \\
Q_{i}^{21} & Q_{i}^{22} & 0 \\
0 & 0 & 1
\end{array}\right]\left(\left[\begin{array}{c}
x_{0} \\
0 \\
1
\end{array}\right]+\left[\begin{array}{c}
Z_{i}^{13} \\
Z_{i}^{23} \\
0
\end{array}\right]\right)+\left[\begin{array}{c}
F_{i}^{13} \\
0 \\
0
\end{array}\right] .
\end{aligned}
$$


Multiplying both sides of the above equation with $\left[\begin{array}{lll}0 & 1 & 0\end{array}\right]$ on the left, we get

$$
\begin{aligned}
& {\left[\begin{array}{lll}
0 & 1 & 0
\end{array}\right]\left[\begin{array}{c}
x_{i+1} \\
x_{i} \\
1
\end{array}\right]=\left[\begin{array}{lll}
0 & 1 & 0
\end{array}\right]\left(\left[\begin{array}{ccc}
Q_{i}^{11} & Q_{i}^{12} & 0 \\
Q_{i}^{21} & Q_{i}^{22} & 0 \\
0 & 0 & 1
\end{array}\right]\left(\left[\begin{array}{c}
x_{0} \\
0 \\
1
\end{array}\right]+\left[\begin{array}{c}
Z_{i}^{13} \\
Z_{i}^{23} \\
0
\end{array}\right]\right)+\left[\begin{array}{c}
F_{i}^{13} \\
0 \\
0
\end{array}\right]\right)} \\
& x_{i}=\left[\begin{array}{lll}
Q_{i}^{21} & Q_{i}^{22} & 0
\end{array}\right]\left(\left[\begin{array}{c}
x_{0} \\
0 \\
1
\end{array}\right]+\left[\begin{array}{c}
Z_{i}^{13} \\
Z_{i}^{23} \\
0
\end{array}\right]\right)+0 \\
& =\left[\begin{array}{ll}
Q_{i}^{21} & Q_{i}^{22}
\end{array}\right]\left(\left[\begin{array}{c}
x_{0} \\
0
\end{array}\right]+\left[\begin{array}{c}
Z_{i}^{13} \\
Z_{i}^{23}
\end{array}\right]\right)=Q_{i-1}^{\prime \prime}\left[\begin{array}{c}
x_{0}+Z_{i}^{13} \\
Z_{i}^{23}
\end{array}\right] \text {. }
\end{aligned}
$$

\subsection{Shared-memory parallel prefix}

A parallel prefix problem on an array $X$ of length $n$ over a binary, associative operator $\odot$ is to determine an array $P$ of length $n$ such that $P[k]=X[0] \odot X[1] \odot X[2] \odot \cdots \odot X[k]$.

This can be done in shared memory for $q$ threads in the following method, known as the 'hypercube' method: 


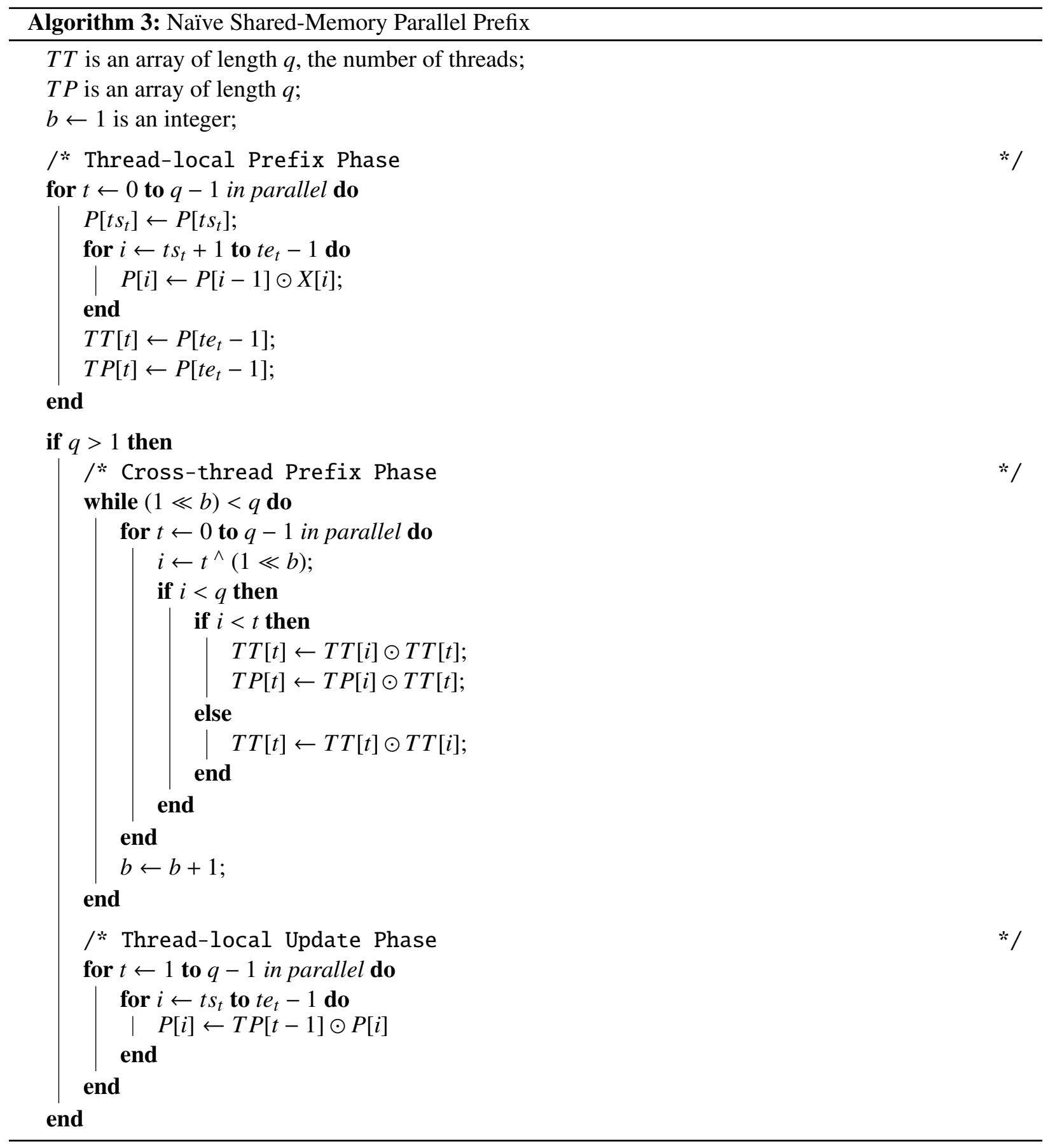

Therefore, where $T_{\odot}$ is the time complexity of the implementation of the $\odot$ operator, and $M_{X}$ is the number of block cells in each element of $X$, the time complexity of Algorithm 3 is

$$
T_{n p p}(n, q)=\left\{\begin{array}{ll}
2\left(\frac{n}{q}+\log _{2}(q)\right) T_{\odot}+2 C_{\text {copy }}\left(q M_{X}\right) & q>1 \\
n T_{\odot}+2 C_{\text {copy }}\left(M_{X}\right) & q=1
\end{array} .\right.
$$


The memory complexity of Algorithm 3 is

$$
M_{n p p}(n, q)=2 C_{\text {cell }} M_{X}(n+q) .
$$

At every stage, the values in $T T$ are available somewhere in $T P$, so we can preclude $T T$ entirely by modifying the Cross-thread Prefix Phase.

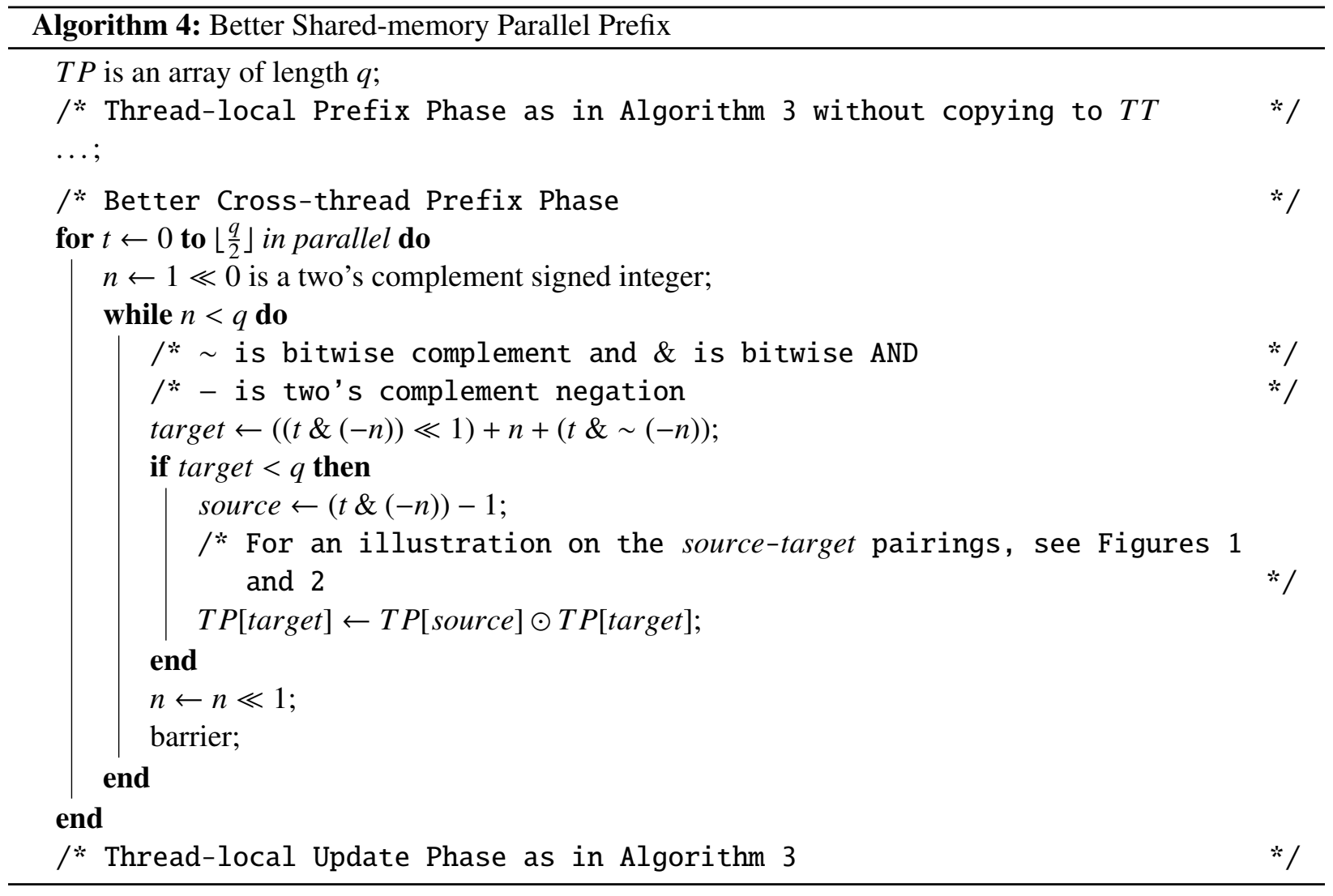

Since this algorithm eliminates the need to copy values to $T T$, this reduces the time complexity to

$$
T_{b p p}(n, q)=\left\{\begin{array}{ll}
\left(2 \frac{n}{q}+\log _{2}(q)\right) T_{\odot}+C_{\text {copy }}\left(q M_{X}\right) & q>1 \\
n T_{\odot}+C_{\text {copy }} M_{X} & q=1
\end{array} .\right.
$$

Since the array $T T$ is eliminated, the memory complexity is reduced to

$$
M_{b p p}(n, q)=C_{\text {cell }} M_{X}(2 n+q) .
$$

\subsubsection{Optimizations for in-place prefix operations}

The prefix scan in the Right-hand side Dependent Phase is a prefix sum of the last column of $Q_{i}^{-1} F_{i}$ for all $i$, in order to determine $Z_{i}^{\prime}$. In this case, $X$ is the array of the last columns of all $Q_{i}^{-1} F_{i}$, and the operation $\odot$ is vector addition. Vector addition can be performed in place.

However, the specific requirements of the Right-hand Side Dependent Phase of the Accelerated Recursive Doubling Algorithm causes Algorithm 3 to have the following drawbacks: 


\section{Figure 1. In-place Shared-Memory Parallel Prefix}

Calculating the prefix sum of the first 24 natural numbers using 8 threads using in-place addition In-place Shared-Memory Parallel Prefix

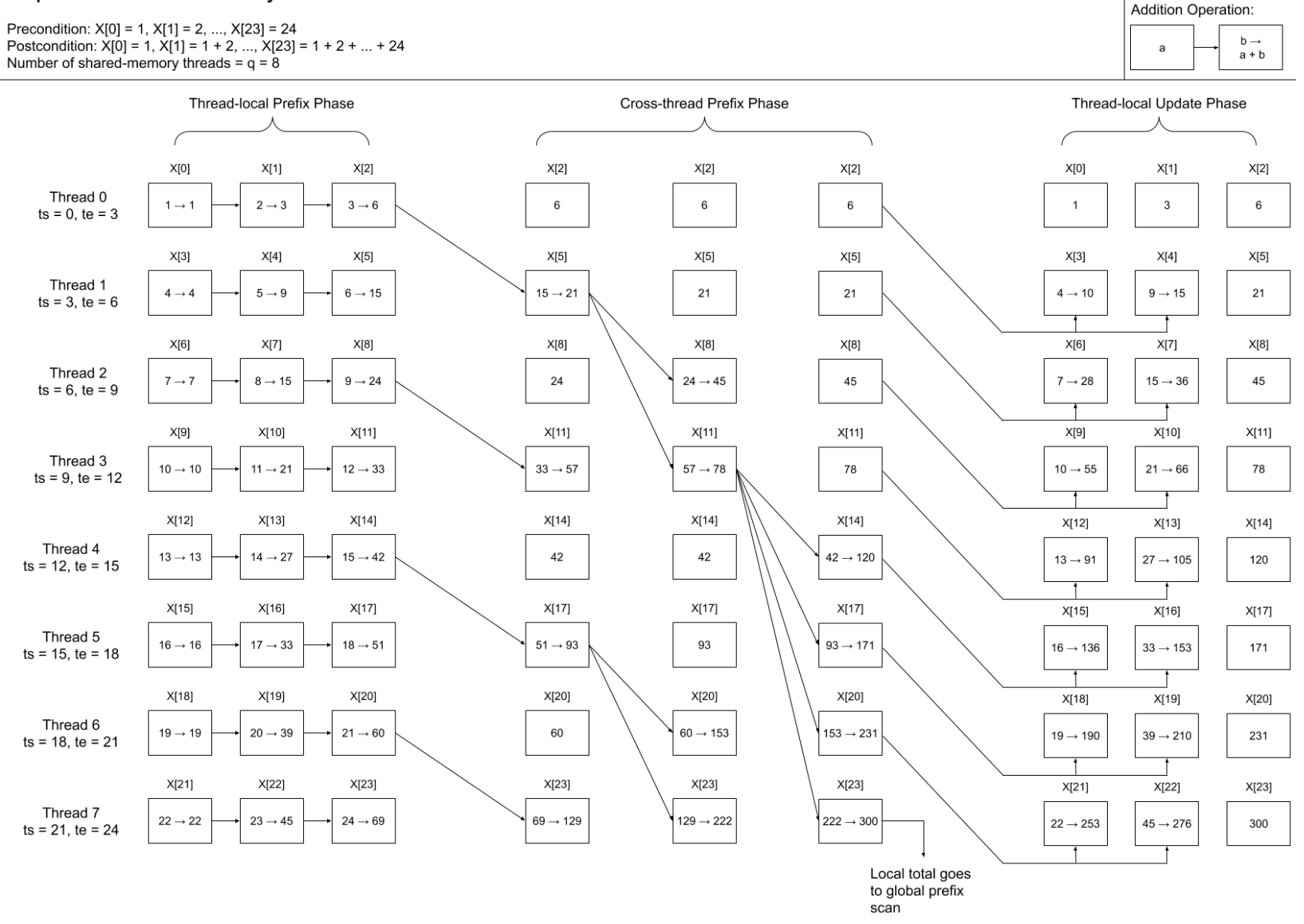

1. The parallel prefix algorithms in ARDA do not require $X$ to be preserved, while the algorithm preserves $X$ and $P$.

2. The algorithm does not take advantage of the fact that the operation $\odot$ is in-place.

These drawbacks can be mitigated by modifying Algorithm 3 as shown in Figure 1 for in-place operations. Figure 1 shows the parallel prefix algorithm for in-place addition on the first 24 natural numbers. The following modifications can be made:

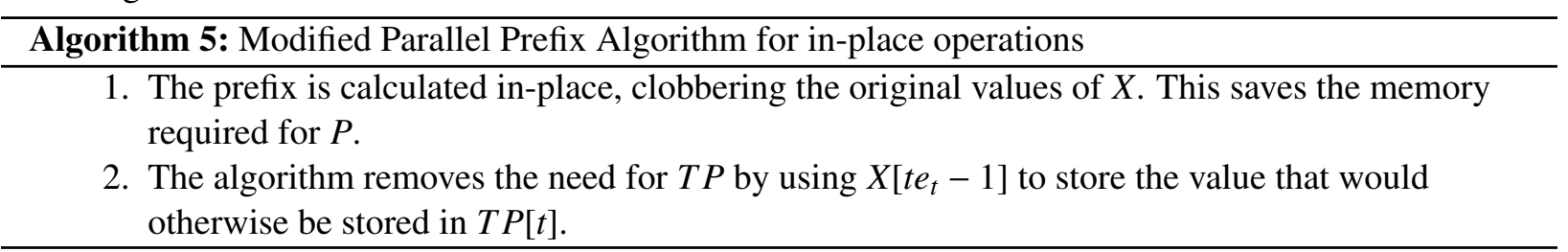


Since this modification eliminates the need to copy values to $T P$, this reduces the time complexity further to

$$
T_{i p p}(n, q)=\left\{\begin{array}{ll}
\left(2 \frac{n}{q}+\log _{2}(q)\right) T_{\odot} & q>1 \\
n T_{\odot} & q=1
\end{array} .\right.
$$

Since the arrays $P$ and $T P$ is eliminated, the memory complexity is reduced to

$$
M_{i p p}(n, q)=n C_{\text {cell }} M_{X} .
$$

Note on threads: The number of concurrent threads running during the Cross-thread Prefix Phase is reduced to $\left\lfloor\frac{q}{2}\right\rfloor$. This means that thread synchronization is faster, and more memory bandwidth is available to the prefix operations.

The number of threads performing during the Thread-local Update Phase is reduced to $q-1$. Since the local total available at $X\left[t e_{q-1}-1\right]$, the $q$-th thread can be assigned to performing the cross-rank parallel prefix operation, providing for some computation-communication overlap.

\subsubsection{Optimizations for out-of-place prefix operations}

The prefix scan in the Right-hand side Independent Phase is a prefix product of $C_{i}^{\prime}$ over all $i$ to determine $Q_{i}^{\prime \prime}$. In this case, $X$ is the array of $C_{i}^{\prime}$, and the operation $\odot$ is matrix multiplication. Matrix multiplication, as implemented in BLAS _ gemm routines, is performed out of place, unlike vector addition.

To make the algorithm behave like as the operation was in place, we could store the result of the matrix multiplication in auxiliary memory and then copy the result, but one copy per operation can be expensive as the size of the matrix increases.

Also, we cannot use $X$ as a substitute for $T P$ as in the case of the out-of-place prefix operations. The cross-thread prefix phase requires multiplying $Q^{\prime}$-type (single-prime) matrices, which are inferred from $Q^{\prime \prime}$-type (double-prime) or $C^{\prime}$-type stored in $X$.

However, we have two thread registers that can store $q$ matrices of type $Q^{\prime}$, and we can use both $R^{01}$ and $R^{34}$ to store $Q^{\prime \prime}$ type registers. Therefore, we can make the following modifications to the algorithm, illustrated in Figure 2 and described below.

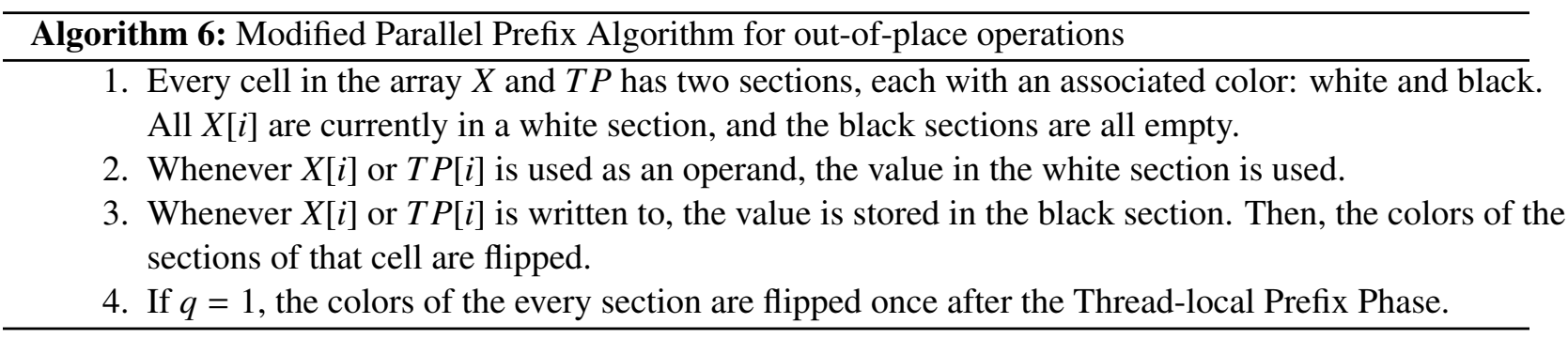

The copy between $X$ and $T P$ in Figure 2 can be eliminated by referring to $X\left[t e_{t}-1\right]$ for the value at $T P[t]$ until $T P[t]$ is written to.

The time complexity of the algorithm is therefore:

$$
T_{\text {oopp }}(n, q)=\left\{\begin{array}{ll}
\left(2 \frac{n}{q}+\log _{2}(q)\right) T_{\odot} & q>1 \\
n T_{\odot} & q=1
\end{array} .\right.
$$


Figure 2. Out-of-place Shared-Memory Parallel Prefix

Calculating the prefix sum of the first 12 natural numbers using 4 threads using out-of-place addition Out-of-place Shared-Memory Parallel Prefix

Precondition: $X[0][$ white $]=1, X[1][$ white $]=2, \ldots, X[11][$ white $]=12$

Postcondition: $X[0][$ white $]=1, X[1][$ white $]=1+2, \ldots, X[11][$ white $]=1+2+\ldots+12$ Number of shared-memory threads $=q=4$

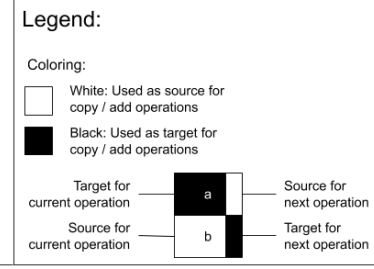

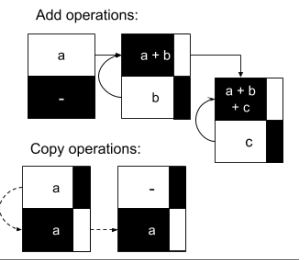

Thread-local Update Phase

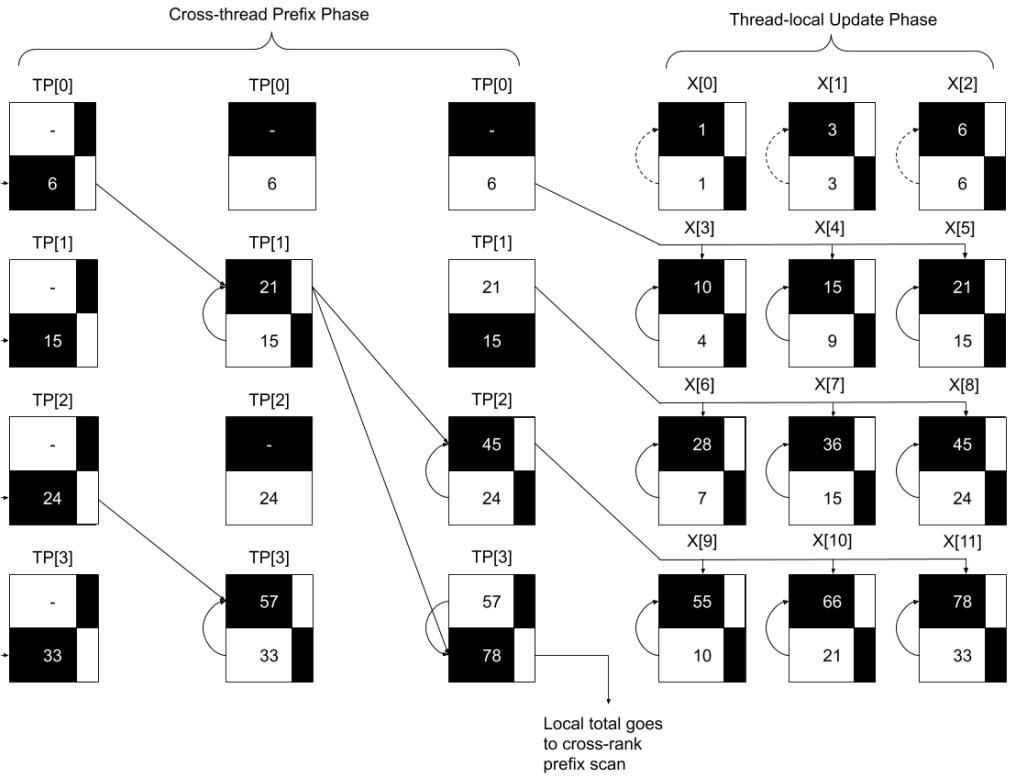


The memory complexity is

$$
M_{\text {oopp }}=2 C_{\text {cell }} M_{X}(n+q) .
$$

Note on threads: Like in the in-place case, the number of concurrent threads running during the Cross-thread Prefix Phase is reduced to $\left\lfloor\frac{q}{2}\right\rfloor$. However, the number of threads performing during the Thread-local Update Phase remains $q$. But, as in Figure 2, Thread 0 is expected to finish early since copying is faster than performing operations like matrix multiplications. Thread 0 can then perform the cross-rank parallel prefix for some computation-communication overlap.

\section{Program}

\subsection{Independent Phase Part 1}

In the first part of the Independent Phase, we have received $L_{i}, D_{i}$, and $U_{i}$, and we calculate $C_{i}^{\prime}$. For any given $k$, the calculation of $C_{k}^{\prime}$ only relies on $L_{k}, D_{k}$, and $U_{k}$. Therefore, we can complete this part using an embarrassingly parallel algorithm as shown in Algorithm 9.

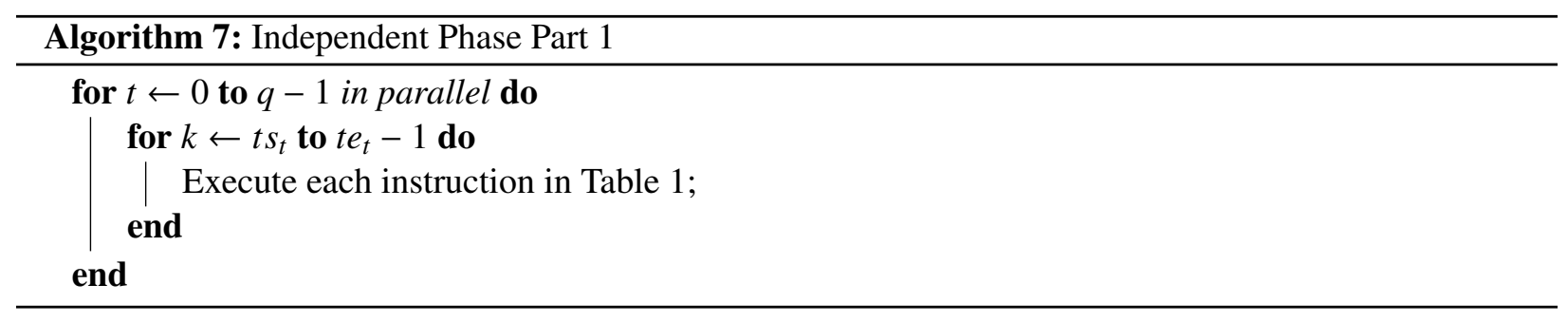

Table 1. Instructions for Independent Phase Part 1

\begin{tabular}{lccccccc}
\hline Instruction & $R_{k}^{0}$ & $R_{k}^{1}$ & $R_{k}^{2}$ & $R_{k}^{3}$ & $R_{k}^{4}$ & $R_{k}^{t 0}$ & Complexity \\
\hline- & $L_{i}$ & $D_{i}$ & $U_{i}$ & - & - & - & - \\
$R_{k}^{2} \leftarrow \operatorname{inv}\left(R_{k}^{2}, R_{t}^{t 0}\right)$ & $L_{i}$ & $D_{i}$ & $U_{i}^{-1}$ & - & - & - & $C_{\mathrm{inv}} M^{3}$ \\
$R_{k}^{34}[0] \leftarrow-R_{k}^{2} R_{k}^{1}$ & $L_{i}$ & $D_{i}$ & $U_{i}^{-1}$ & $\left(C_{i}^{11},-\right)$ & - & $C_{\mathrm{mul}} M^{3}$ \\
$R_{k}^{34}[1] \leftarrow-R_{k}^{2} R_{k}^{0}$ & $L_{i}$ & $D_{i}$ & $U_{i}^{-1}$ & $\left(C_{i}^{11}, C_{i}^{12}\right)=C_{i}^{\prime}$ & - & $C_{\mathrm{mul}} M^{3}$ \\
\hline
\end{tabular}

The time complexity of first part of the independent phase is

$$
T_{i p 1}(M, N, P, q)=\frac{N}{P q}\left(M^{3}\left(C_{\mathrm{inv}}+2 C_{\mathrm{mul}}\right)\right) .
$$




\subsection{Independent Phase Part 2}

In the second part of the Independent Phase, we have $C_{i}^{\prime}$, and we must calculate $Q_{i}^{\prime \prime}$. This can be done efficiently using a parallel prefix scan. The prefix scan has the following parts:

1. Compute the shared-memory prefix product of all $C_{i}^{\prime}$ on a given processing element to obtain 'local' $Q_{i}^{\prime \prime}$.

2. Compute the distributed-memory prefix product of the 'local' $Q_{i}^{\prime}$ 's that represent the local total.

3. Update the 'local' $Q_{i}^{\prime \prime}$ using the result of the previous step to get $Q_{i}^{\prime \prime}$.

Step 1 is implemented using Algorithm 6. The sections of $X[i]$ are $R^{34}[i]$ and $R^{01}[i]$ with the $R^{34}$ section initially colored white. The sections of $T P[i]$ are $R^{t 0}[i]$ and $R^{t 1}[i]$ with the $R^{t 0}$ section initially colored white.

For the particular application of calculating the prefix sum of $C_{i}$ matrices, we can further optimize the storage and computation time for the parallel prefix algorithm. In the Thread-local Prefix and the Thread-local Update stages, the resultant matrices are products of collections of $C_{i}$ matrices. This means we can omit calculating the second block rows of the resultant matrices, and infer the second block rows from previous matrices. This means that the cost of the prefix operation is not uniform over the prefix operation.

This means Equation (22) cannot be used to express the time complexity of this step. The time complexity can be calculated to be:

$$
\begin{aligned}
T_{i p 21}(M, N, P, q) & =T_{C^{\prime} \times Q^{\prime \prime}}(M) \frac{N}{P q}+ \begin{cases}T_{Q^{\prime} \times Q^{\prime}}(M) \log _{2}(q)+T_{Q^{\prime \prime} \times Q^{\prime}}(M) \frac{N}{P q} & q>1 \\
0 & q=1\end{cases} \\
& =M^{3} C_{\mathrm{mul}}\left\{\begin{array}{ll}
8 \frac{N}{p q}+8 \log _{2}(q) & q>1 \\
4 \frac{N}{q} & q=1
\end{array} .\right.
\end{aligned}
$$

Step 2 is implemented using a distributed-memory library function like MPI_Exscan using a simulated in-place matrix multiplication operator, which first multiplies two matrix into some auxiliary storage $\left(R_{0}^{t 0}\right)$ and copies the result into the memory held by an operand. The result is received in the receive register $R^{r}$. The time complexity of this step is therefore:

$$
T_{i p 22}(M, N, P, q)=\left(M^{3}\left(8 C_{\text {mul }}\right)+M^{2}\left(4 C_{\text {copy }}\right)\right) \log _{2}(P)+\left(\tau+4 \mu M^{2}\right) \log _{2}(P) .
$$

The $4 M^{2} C_{\text {copy }}$ term can be eliminated by using a parallel prefix method optimized for out-of-place operations like in Step 1. However, this was not done in this study because the convenience of using a library function outweighed the potential gain by the complex optimization.

Step 3 can be written as an embarrassingly parallel algorithm as given in Algorithm 8.

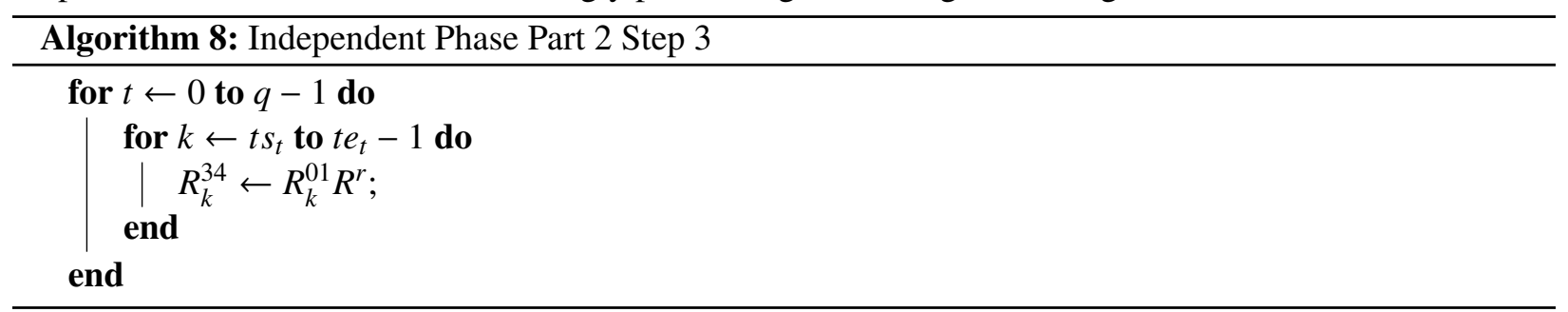


Therefore, the time complexity of this step

$$
T_{i p 23}(M, N, P, q)=M^{3}\left(4 \frac{N}{P q} C_{\mathrm{mul}}\right) .
$$

The time complexity of the second part of the independent phase is

$$
\begin{aligned}
T_{i p}(M, N, P, q)= & M^{2}\left(4 C_{\mathrm{copy}}+4 \mu\right) \log _{2} P+\tau \log _{2} P+ \\
& M^{3}\left(8 C_{\mathrm{mul}} \log _{2} P+\left\{\begin{array}{ll}
\frac{N}{P q}\left(12 C_{\mathrm{mul}}\right)+8 C_{\mathrm{mul}} \log _{2} q & q>1 \\
\frac{N}{P}\left(8 C_{\mathrm{mul}}\right) & q=1
\end{array}\right) .\right.
\end{aligned}
$$

\subsection{Independent Phase Part 3}

This phase can be implemented using an embarrasingly parallel algorithm as follows:

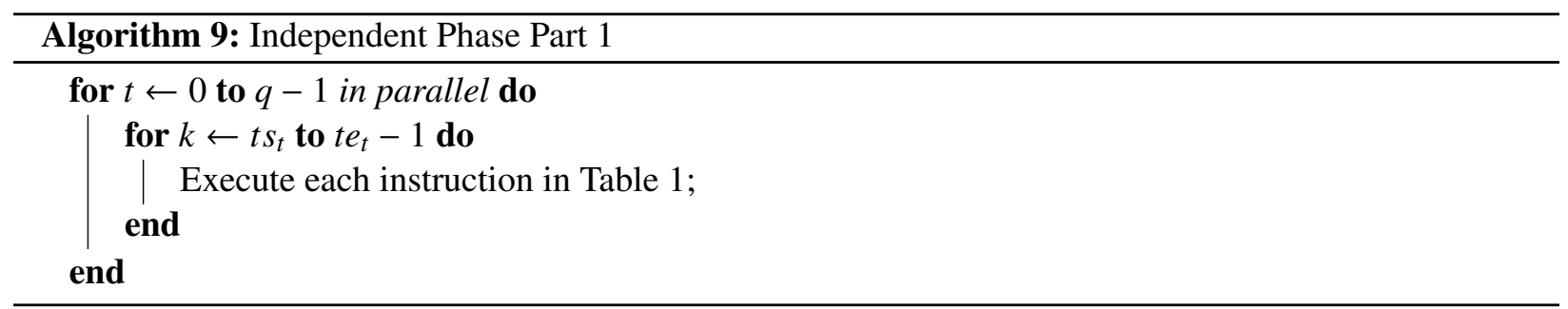

In the following table, $\operatorname{inv}\left(R^{A}, R^{B}\right)$ is a function that inverts the matrix stored in $R^{A}$, in place, using $R^{B}$ as a workspace.

Table 2. Instructions for Independent Phase Part 3

\begin{tabular}{llllll}
\hline Instruction & $R_{k}^{0}$ & $R_{k}^{1}$ & $R_{k}^{t 0}$ & $R_{k}^{t 1}$ & Complexity \\
\hline$R_{t}^{t 0} \leftarrow R_{K-k}^{34}[0]$ & - & - & $A$ & - & $C_{\text {copy }} M^{2}$ \\
$R_{t}^{t 0} \leftarrow \operatorname{inv}\left(R_{k}^{t 0}, R_{t}^{t 1}\right)$ & - & - & $A^{-1}$ & - & $C_{\text {inv }} M^{3}$ \\
$R_{k}^{0} \leftarrow R_{K-k+1}^{34}[0] R_{t}^{t 0}$ & $C A^{-1}$ & - & $A^{-1}$ & - & $C_{\text {mul }} M^{3}$ \\
$R_{t}^{t 1} \leftarrow R_{K-k+1}^{34}[1]$ & $C A^{-1}$ & - & $A^{-1}$ & $D$ & $C_{\text {copy }} M^{2}$ \\
$R_{t}^{t 1} \leftarrow R_{t}^{t 1}-R_{k}^{0} R_{K-i}^{34}[1]$ & $C A^{-1}$ & - & $A^{-1}$ & $V$ & $C_{\text {mul }} M^{3}$ \\
$R_{t}^{t 1} \leftarrow i n v\left(R_{t}^{t 1}, R_{k}^{1}\right)$ & $C A^{-1}$ & - & $A^{-1}$ & $V^{-1}$ & $C_{\text {inv }} M^{3}$ \\
$R_{k}^{1} \leftarrow-R_{t}^{t 0} R_{k}^{0}$ & $C A^{-1}$ & $-V^{-1} C A^{-1}$ & $A^{-1}$ & $V^{-1}$ & $C_{\mathrm{mul}} M^{3}$ \\
$R_{t}^{t 1} \leftarrow-R_{K-k}^{34}[1] R_{k}^{1}$ & $C A^{-1}$ & $-V^{-1} C A^{-1}$ & $A^{-1}$ & $W$ & $C_{\mathrm{mul}} M^{3}$ \\
$R_{t}^{t 1} \leftarrow R_{t}^{t 1}+I$ & $C A^{-1}$ & $-V^{-1} C A^{-1}$ & $A^{-1}$ & $I+W$ & $C_{\mathrm{add}} M$ \\
$R^{0} \leftarrow R_{t}^{t 0} R_{t}^{t 1}$ & $A^{-1}(I+W)$ & $-V^{-1} C A^{-1}$ & $A^{-1}$ & $I+W$ & $C_{\mathrm{mul}} M^{3}$ \\
\hline
\end{tabular}


The final results in $R_{k}^{0}$ and $R_{k}^{1}$ form the first column of the inverse of $Q_{i}$, as described in the Optimizations in the Independent Phase section.

The time complexity of the third part of the independent phase is

$$
T_{i p 3}(M, N, P, q)=\left(M^{3}\left(2 C_{\mathrm{inv}}+5 C_{\mathrm{mul}}\right)+M^{2}\left(2 C_{\text {copy }}\right)+M\left(C_{\text {add }}\right)\right) \frac{N}{P q} .
$$

\subsection{Dependent Phase Part 1}

In the first part of the Dependent Phase, we have $b_{i}, U_{i}^{-1}$, and the first column of $Q_{i}^{-1}$ and we calculate $z_{i}$. For any given $k$, the calculation of $z_{k}$ only relies on $b_{i}, U_{k}^{-1}$, and the first column of $Q_{k}^{-1}$. Therefore, we can implement this part using an embarrassingly parallel algorithm as shown in Algorithm 10.

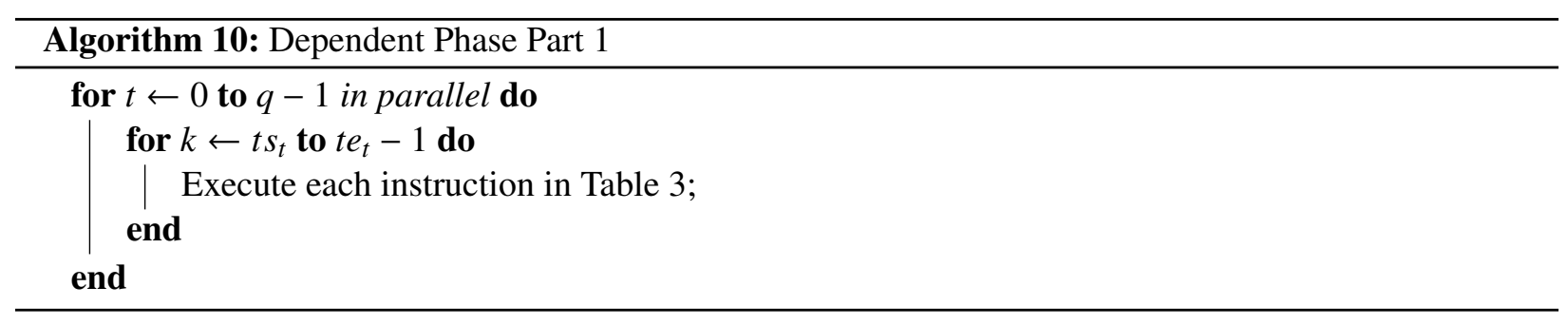

Table 3. Instructions for Dependent Phase Part 1

\begin{tabular}{llccc}
\hline Instruction & $R_{k}^{v 0}$ & $R_{k}^{v 1}$ & $R_{k}^{\nu 2}$ & Complexity \\
\hline- & $b_{i}$ & - & - & - \\
$R_{k}^{\nu 2} \leftarrow R^{2} R^{v 0}$ & $b_{i}$ & - & $F_{i}^{13}$ & $C_{\mathrm{mul}} M^{2}$ \\
\cline { 2 - 3 }$R_{k}^{Z}[0] \leftarrow R_{k}^{0} R_{k}^{v 2}$ & $\left(\left(Q_{i}^{-1} F_{i}\right)^{13},-\right)$ & $F_{i}^{13}$ & $C_{\mathrm{mul}} M^{2}$ \\
$R_{k}^{Z}[1] \leftarrow R_{k}^{1} R_{k}^{v 2}$ & $\left(\left(Q_{i}^{-1} F_{i}\right)^{13},\left(Q_{i}^{-1} F_{i}\right)^{23}\right)=z_{i}$ & $F_{i}^{13}$ & $C_{\mathrm{mul}} M^{2}$ \\
\hline
\end{tabular}

The time complexity of this part of the algorithm is

$$
T_{d p 1}(N, M, P, q)=\frac{N}{P q}\left(M^{2}\left(3 C_{\mathrm{mul}}\right)\right)
$$

\subsection{Dependent Phase Part 2}

In the second part of the Dependent Phase, we have $z_{i}$, and we must calculate $Z_{i}^{\prime}$. This can be done using a parallel prefix scan. The prefix scan has the following parts:

1. Compute the shared-memory prefix sum of all $z_{i}$ to obtain 'local' $Z_{i}$.

2. Compute the distributed-memory prefix sum of the 'local' $Z_{i}$ 's that represent the local totals. 
3. Update the 'local' $Z_{i}$ using the result of the previous step to get $Z_{i}^{\prime}$.

4. On the processing element that has $Q_{N}$, calculate $x_{1}=-\left(Q_{N}^{11}\right)^{-1} F_{N}$ and broadcast it to all processing elements.

Step 1 is implemented using Algorithm 5. The register $R^{Z}$ is represented by $X$.

Substituting $T_{\odot}=M\left(2 C_{\text {add }}\right), n=\frac{N}{P}$ in Equation (20), we get

$$
T_{d p 21}(M, N, P, q)=\left\{\begin{array}{ll}
M\left(2 \frac{N}{P q}+\log _{2}(q)\right)\left(2 C_{\text {add }}\right) & q>1 \\
M\left(\frac{N}{P}\left(2 C_{\text {add }}\right)\right) & q=1
\end{array} .\right.
$$

Step 2 is implemented using a distributed-memory library function like MPI_Exscan using a vector addition operator, where the result is received in $R_{v 0}^{t 0}$. The time complexity of this step is therefore:

$$
T_{d p 22}(M, N, P, q)=M\left(2 C_{\text {add }}\right) \log _{2}(P)+(\tau+2 \mu M) \log _{2}(P) .
$$

Step 3 can be implemented as an embarrassingly parallel algorithm as given in Algorithm 11.

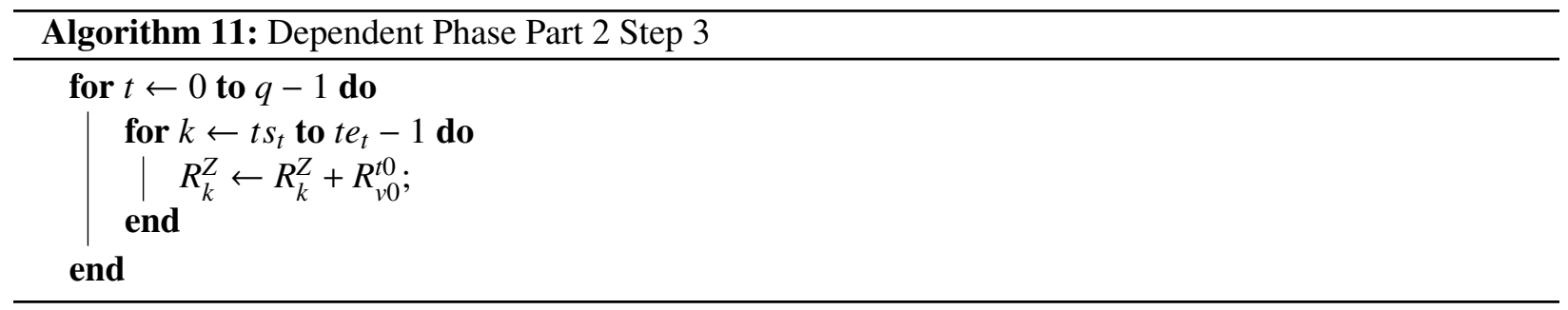

Therefore, the time complexity of this step is

$$
T_{d p 23}(M, N, P, q)=M\left(\frac{N}{P q} C_{\text {add }}\right) .
$$

Step 4 can be implemented as in Algorithm 12.

\begin{tabular}{l}
\hline Algorithm 12: Dependent Phase Part 2 Step 4 \\
\hline if $N$ is on current processing element then \\
$\mid \begin{array}{l}R^{t 1}[0]^{11} \leftarrow \operatorname{inv}\left(R^{34}\left[K_{r}-1\right], R^{t 1}[0]^{21}\right) ; \\
R_{v 1}^{t 0} \leftarrow-R^{t 1}[0]^{11} R_{K_{r}-1}^{v 2} ;\end{array}$ \\
end \\
Broadcast $R_{v 1}^{t 0}$, receiving in $R_{v 1}^{t 0} ;$
\end{tabular}

The time complexity of this step is

$$
T_{d p 24}(M, N, P, q)=C_{\mathrm{inv}} M^{3}+C_{\mathrm{mul}} M^{2}+(\tau+\mu M) \log _{2} P .
$$

The total time complexity of the second part of the dependent phase is therefore

$$
T_{d p}(M, N, P, q)=\tau \log _{2}(P)+M\left(2\left(C_{\text {add }}+\mu\right) \log _{2}(P)+\left\{\begin{array}{ll}
\left.\frac{N}{P q}\left(5 C_{\text {add }}\right)+2 C_{\text {add }} \log _{2}(q)\right) & q>1 \\
\frac{N}{P}\left(3 C_{\text {add }}\right) & q=1
\end{array}\right) .\right.
$$




\subsection{Dependent Phase Part 3}

In the third part of the Dependent Phase, we have $Z_{i}^{\prime}, x_{1}$, and $Q_{i}^{\prime \prime}$, and we must calculate $x_{i}$. For any given $k$, the calculation of $x_{k}$ only relies on $Z_{k}^{\prime}, x_{1}$, and $Q_{k-1}^{\prime \prime}$. Therefore, we can implement this part using an embarrassingly parallel algorithm as shown in Algorithm 13.

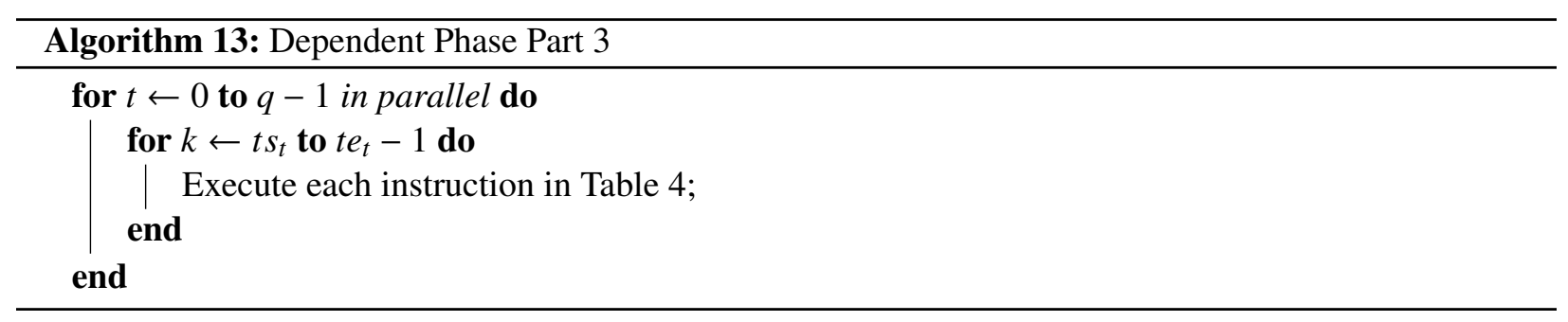

Table 4. Instructions for Dependent Phase Part 3

\begin{tabular}{llcc}
\hline Instruction & $R_{k}^{Z}$ & $R_{k}^{v 2}$ & Complexity \\
\hline- & $Z_{i}^{\prime}$ & $F_{i}^{13}$ & - \\
$R_{k}^{Z} \leftarrow R_{k}^{Z}+\left[\begin{array}{ll}x_{1} & 0\end{array}\right]^{T}$ & $Z_{i}^{\prime}+\left[\begin{array}{ll}x_{1} & 0\end{array}\right]^{T}$ & $F_{i}^{13}$ & $C_{\text {add }} M$ \\
$R_{k}^{v 2} \leftarrow R_{k}^{v 2}+R_{K-i+1}^{34} R_{k-1}^{Z}$ & $Z_{i}^{\prime}+\left[\begin{array}{ll}x_{1} & 0\end{array}\right]^{T}$ & $x_{i}$ & $2 C_{\text {mul }} M^{2}$ \\
\hline
\end{tabular}

The time complexity of this part of the algorithm is

$$
T_{d p 3}(M, N, P, q)=\frac{N}{P q}\left(M^{2}\left(2 C_{\mathrm{mul}}\right)+M C_{\mathrm{add}}\right) .
$$

\section{Time Complexity Analysis and Comparison}

\subsection{Naïve implementation}

The naïve implementation is considered to use $M \times M$ matrices to represent $L, D$ and $U$ matrices, $2 M \times 2 M$ matrices to represent $C$ and $Q$ matrices, while using $2 M \times 1$ matrices to represent $F$ and $Z$ matrices.

To determine the time complexity of the dependent phase of the naïve implementation, we list the time-complexities of the naïve implementations of each step, and then sum the complexities. 


\subsubsection{Independent phase}

\begin{tabular}{ll}
\hline Step & Complexity \\
\hline Invert $U$ 's & $\frac{N}{P}\left(C_{\text {inv }} M^{3}\right)$ \\
Calculate $C$ 's using $L, D$, and $U^{-1}$ & $\frac{N}{P}\left(2 C_{\mathrm{mul}} M^{3}\right)$ \\
Prefix $C$ 's locally to find local $Q$ 's & $\frac{N}{P}\left(8 C_{\mathrm{mul}} M^{3}\right)$ \\
Cross-rank prefix scan on $Q$ & $\left(\tau+4 \mu M^{2}+8 C_{\mathrm{mul}} M^{3}\right) \log _{2}(P)$ \\
Update $Q$ 's locally to find global $Q$ 's & $\frac{N}{P}\left(8 C_{\mathrm{mul}} M^{3}\right)$ \\
Invert $Q$ 's & $\frac{N}{P}\left(8 C_{\mathrm{inv}} M^{3}\right)$ \\
\hline
\end{tabular}

The total time complexity for the independent phase is

$$
\left.T_{n i}(M, N, P)=M^{3}\left(\frac{N}{P}\left(9 C_{\mathrm{inv}}+18 C_{\mathrm{mul}}\right)\right)+8 C_{\mathrm{mul}} \log _{2}(P)\right)+\Theta\left(M^{2} \log _{2} P\right)
$$

\subsubsection{Dependent Phase}

\begin{tabular}{ll}
\hline Step & Complexity \\
\hline Calculate $F$ 's & $\frac{N}{P}\left(C_{\mathrm{mul}} M^{2}\right)$ \\
Calculate $V$ 's & $\frac{N}{P}\left(2 C_{\mathrm{mul}} M^{2}\right)$ \\
Prefix $V$ 's locally to find local $Z$ 's & $\frac{N}{P}\left(2 C_{\mathrm{add}} M\right)$ \\
Cross-rank prefix scan on $Z$ & $\left(\tau+2 \mu M+2 C_{\mathrm{add}} M\right) \log _{2} P$ \\
Update $Z$ 's locally to find global $Z$ 's & $\frac{N}{P}\left(2 C_{\mathrm{add}} M\right)$ \\
Calculate $E$ 's using $Z, Q$ and $F$ & $\frac{N}{P}\left(2 C_{\mathrm{mul}} M^{2}+2 C_{\mathrm{add}} M\right)$ \\
Calculate $x_{1}$ & $C_{\mathrm{inv}} M^{3}+C_{\mathrm{mul}} M^{2}$ \\
Broadcast $x_{1}$ & $(\tau+\mu M) \log _{2} P$ \\
Calculate $x_{i}$ using $x_{0}, Q$, and $E$ & $\frac{N}{P} C_{\mathrm{mul}}\left(4 M^{2}+2 M\right)$ \\
\hline
\end{tabular}

The total time complexity for the dependent phase is

$$
T_{n d}(M, N, P)=M^{3} C_{\mathrm{inv}}+M^{2}\left(\frac{N}{P}\left(9 C_{\mathrm{mul}}\right)+C_{\mathrm{mul}}\right)+\Theta\left(\frac{M N}{P}+M \log _{2} P\right)
$$




\subsection{Optimized implementation}

\subsubsection{Independent phase}

Summing up the time complexities given in Equations (24), (29), and (30), we get:

$$
\begin{aligned}
T_{o i}(M, N, P, q)= & M^{3}\left(8 C_{\mathrm{mul}} \log _{2} P+\frac{N}{P q}\left(3 C_{\mathrm{inv}}\right)+\left\{\begin{array}{ll}
\frac{N}{P q} 19 C_{\mathrm{mul}}+8 C_{\mathrm{mul}} \log _{2} q & q>1 \\
\frac{N}{P} 15 C_{\mathrm{mul}} & q=1
\end{array}\right)+\right. \\
& M^{2}\left(\frac{N}{P q}\left(2 C_{\mathrm{copy}}\right)+\left(4 C_{\mathrm{copy}}+4 \mu\right) \log _{2} P\right)+M \frac{N}{P q} C_{\mathrm{add}} \\
= & M^{3}\left(8 C_{\mathrm{mul}} \log _{2} P+\frac{N}{P q}\left(3 C_{\mathrm{inv}}\right)+\left\{\begin{array}{ll}
\frac{N}{P q} 19 C_{\mathrm{mul}}+8 C_{\mathrm{mul}} \log _{2} q & q>1 \\
\frac{N}{P} 15 C_{\mathrm{mul}} & q=1
\end{array}\right)+\right. \\
& \Theta\left(\frac{M^{2} N}{P q}+M^{2} \log _{2} P\right) .
\end{aligned}
$$

Setting $q=1$, we get

$$
T_{o i}(M, N, P)=M^{3}\left(\frac{N}{P}\left(15 C_{\mathrm{mul}}+3 C_{\mathrm{inv}}\right)+8 C_{\mathrm{mul}} \log _{2} P\right)+\Theta\left(\frac{M^{2} N}{P}+M^{2} \log _{2} P\right)
$$

\subsubsection{Dependent phase}

Summing up the time complexities given in Equations (31), (36), and (31), we get:

$$
\begin{aligned}
T_{o d}(M, N, P, q)= & M^{3} C_{\mathrm{inv}}+M^{2}\left(\frac{N}{P q}\left(5 C_{\mathrm{mul}}\right)+C_{\mathrm{mul}}\right)+ \\
& 2 \tau \log _{2} P+M\left(\left(2 C_{\mathrm{add}}+3 \mu\right) \log _{2} P+2 C_{\mathrm{add}}\left\{\begin{array}{ll}
3 \frac{N}{P q}+\log _{2} q & q>1 \\
2 \frac{N}{P} & q=1
\end{array}\right)\right. \\
= & M^{3} C_{\mathrm{inv}}+M^{2}\left(\frac{N}{P q}\left(5 C_{\mathrm{mul}}\right)+C_{\mathrm{mul}}\right)+\Theta\left(\frac{M N}{P q}+M \log _{2} P+M \log _{2} q\right)
\end{aligned}
$$

Setting $q=1$, we get

$$
T_{o d}(M, N, P)=M^{3} C_{\mathrm{inv}}+M^{2}\left(\frac{N}{P}\left(5 C_{\mathrm{mul}}\right)+C_{\mathrm{mul}}\right)+\Theta\left(\frac{M N}{P}+M \log _{2} P\right)
$$

\subsection{Comparison}

In order to compare the two algorithms, we assume that the number of shared-memory threads is $q=1$, and $C_{\mathrm{inv}}=C_{\mathrm{mul}}=C$. For the two problem parameters $M$ and $N$, we choose the dominantly growing term that grows along with that parameter.

We then measure the asymptotic speedup defined as the limit

$$
\lim _{\text {parameter } \rightarrow \infty} \frac{\text { Dominant term of the naïve implementation }}{\text { Dominant term of the optimized implementation }} \text {. }
$$




\subsubsection{Independent Phase}

\begin{tabular}{llll}
\hline Parameter & Dominant term (Optimized) & Dominant term (Naïve) & Asymptotic Speedup \\
\hline $\mathrm{M}$ & $18 C M^{3} \frac{N}{P}+8 C M^{3} \log _{2} P$ & $27 C M^{3} \frac{N}{P}+8 C M^{3} \log _{2} P$ & $\frac{27 N+8 P \log _{2} P}{18 N+8 P \log _{2} P}=\frac{3}{2}(N>>P)$ \\
$\mathrm{N}$ & $18 C M^{3} \frac{N}{P}$ & $27 C M^{3} \frac{N}{P}$ & $\frac{3}{2}$ \\
\hline
\end{tabular}

\subsubsection{Dependent Phase}

\begin{tabular}{llll}
\hline Parameter & Dominant term (Optimized) & Dominant term (Naïve) & Asymptotic Speedup \\
\hline $\mathrm{M}$ & $C M^{3}$ & $C M^{3}$ & 1 \\
$\mathrm{~N}$ & $5 C M^{2} \frac{N}{P}$ & $9 C M^{2} \frac{N}{p}$ & $\frac{9}{5}$ \\
\hline
\end{tabular}

\section{Memory Complexity Analysis and Comparison}

\subsection{Naïve implementation}

The memory complexity of the naïve implementation was estimated by summing up the sizes of the allocations relevant to the phase of the algorithm over all processing elements in the implementation of the ARDA algorithm used in [2].

For the independent phase, the memory complexity was found to be $M_{n i}(M, N, P) \geq\left(14 M^{2} N+8 M^{2} P\right) C_{\text {cell }}$. For the dependent phase, the memory complexity was found to be $M_{n d}(M, N, P) \geq(6 M N+4 M P) C_{\text {cell }}$.

\subsection{Optimized implementation}

The memory complexity of the optimized implementation can be measured simply by summing up the sizes of the registers relevant to the phase of the algorithm.

For the independent phase, the memory complexity was found to be $M_{o i}(M, N, P, q)=\left(5 M^{2} N+8 M^{2} P q+4 M^{2} P\right) C_{\text {cell }}$. For the dependent phase, the memory complexity was found to be $M_{o d}(M, N, P, q)=(3 M N+3 M P q) C_{\text {cell }}$.

\subsection{Comparison}

In order to compare the two algorithms, we assume that the number of shared-memory threads is $q=1$. For the two problem parameters $M, N$, we choose the dominantly growing term that grows along with that parameter. 
We then measure the asymptotic memory savings defined as the limit

$$
\lim _{\text {parameter } \rightarrow \infty} \frac{\text { Dominant term of the optimized implementation }}{\text { Dominant term of the naïve implementation }} .
$$

\subsubsection{Independent Phase}

\begin{tabular}{llll}
\hline Parameter & Dominant term (Optimized) & Dominant term (Naïve) & Asymptotic Memory Savings \\
\hline $\mathrm{M}$ & $M^{2}(5 N+12 P)$ & $M^{2}(14 N+8 P)$ & $\frac{5 N+12 P}{14 N+8 P}=\frac{5}{14}(N>>P)$ \\
$\mathrm{N}$ & $N\left(5 M^{2}\right)$ & $N\left(14 M^{2}\right)$ & $\frac{5}{14}$ \\
\hline
\end{tabular}

\subsubsection{Dependent Phase}

\begin{tabular}{llll}
\hline Parameter & Dominant term (Optimized) & Dominant term (Naïve) & Asymptotic Memory Savings \\
\hline $\mathrm{M}$ & $M(3 N+3 P)$ & $M(6 N+4 P)$ & $3 \frac{\frac{N}{P}+1}{6 \frac{N}{P}+4}$ \\
$\mathrm{~N}$ & $N(3 N)$ & $N(6 M)$ & $\frac{1}{2}$ \\
\hline
\end{tabular}

\section{Shared-memory threading speedups}

We determine the effect of adding shared-memory threading to the asymptotic time-complexity of the Algorithm.

\subsection{Independent phase}

The asymptotic time complexity of the independent phase is

$$
T_{o i}(M, N, P, q)=\Theta\left(\frac{M^{3} N}{P q}+M^{3} \log _{2}(P q)\right) .
$$

Therefore, the asymptotic speedup with increasing $q$ is

$$
\begin{aligned}
\text { Speedup } & =\frac{\text { Complexity for num threads }=1}{\text { Complexity for num threads }=q}=\Theta\left(\frac{\frac{M^{3} N}{P}+M^{3} \log _{2}(P)}{\frac{M^{3} N}{P q}+M^{3} \log _{2}(P q)}\right) \\
& =\Theta\left(\frac{q\left(\frac{N}{P}+\log _{2}(P)\right)}{\frac{N}{P}+q \log _{2}(P q)}\right) .
\end{aligned}
$$


For small block-row granularities $\frac{N}{P}$, we can plug in $\frac{N}{P} \approx 0$ in the above equation to obtain an asymptotic speedup of $\Theta(1)$. For large block-row granularities, we can plug in $\frac{\frac{N}{P}+\log _{2} P}{\frac{N}{P}} \approx \frac{\frac{N}{P}+q \log _{2}(P q)}{\frac{N}{P}} \approx 1$ to obtain an asymptotic speedup of $\Theta(q)$.

\subsection{Dependent phase}

The asymptotic time complexity of the dependent phase is

$$
T_{o d}(M, N, P, q)=\Theta\left(M^{3}+\frac{M^{2} N}{P q}+M \log _{2}(P q)\right) .
$$

Therefore, the asymptotic speedup with increasing $q$ is

$$
\begin{aligned}
\text { Speedup } & =\frac{\text { Complexity for num threads }=1}{\text { Complexity for num threads }=q}=\Theta\left(\frac{M^{3}+\frac{M^{2} N}{P}+M \log _{2} P}{M^{3}+\frac{M^{2} N}{P q}+M \log _{2}(P q)}\right) \\
& =\Theta\left(\frac{q\left(\frac{M N}{P}+M^{2}+\log _{2} P\right)}{\frac{M N}{P}+q\left(M^{2}+\log _{2}(P q)\right)}\right) .
\end{aligned}
$$

For small block-cell granularities $\frac{M N}{P}$, we can plug in $\frac{M N}{P} \approx 0$ in the above equation to obtain an asymptotic speedup of $\Theta(1)$. For large block-cell granularities, we can plug in $\frac{\frac{M N}{P}+M^{2}+\log _{2} P}{\frac{M N}{P}} \approx \frac{\frac{M N}{P}+q\left(M^{2}+\log _{2}(P q)\right)}{\frac{M N}{P}} \approx 1$ to obtain an asymptotic speedup of $\Theta(q)$.

\section{Experiments and Results}

The optimized algorithm described was implemented in C, using BLAS and LAPACK calls for matrix and vector operations. The solutions generated by the algorithm were measured for accuracy by the error function $E(x)=\log _{2} \frac{\left\|A x^{*}-b\right\|}{M N}$ where $x^{*}$ is the solution given by the algorithm. The smaller the value of $E\left(x^{*}\right)$, the more accurate the solution is.

The results were as follows:

Table 5. Mean error for $M=2, N=20$

\begin{tabular}{|l|l|}
\hline$P$ & Mean $E(x)$ \\
\hline 6 & -22.177 \\
8 & -24.389 \\
10 & -21.317 \\
12 & -21.318 \\
\hline
\end{tabular}

Table 6. Mean error for $M=3, N=9$

\begin{tabular}{|l|l|}
\hline$P$ & Mean $E(x)$ \\
\hline 6 & -21.518 \\
8 & -22.116 \\
10 & -21.232 \\
12 & -25.232 \\
\hline
\end{tabular}

The optimized implementation works correctly, with the magnitude of the error $=2^{E\left(x^{8}\right)} \leq 10^{-7}$ for the above problem sizes. 
However, for larger problem sizes, we get

Table 7. Mean error for $M=3, N=22$

\begin{tabular}{|l|l|}
\hline$P$ & Mean $E(x)$ \\
\hline 6 & 9.020 \\
8 & 9.740 \\
10 & 9.740 \\
12 & 9.087 \\
\hline
\end{tabular}

The error continues to rise rapidly with increases in $M$ and $N$, and $E(x)$ approaches 225 for $N=20$, $M=80$. The rapidly increasing error poses a significant challenge to the practical use of this algorithm. The possible causes have been identified as

1. The blockwise inverting algorithm used in Optimization 2 to efficiently calculate only the first block column

2. The limited precision to which we can store large numbers resulting from prefix products of large numbers of matrices.

New techniques need to be developed to keep the error within practical limits.

\section{Conclusions}

We presented alternative mathematical formulations of intermediary matrices in the algorithm. We also made the assumptions made by the algorithm explicit. This helped us determine that a particular algorithm cited in [2] will not run.

We have shown methods by which the Accelerated Recursive Doubling Algorithm can be optimized, and shown how the speedup and memory savings is affected by the value of the problem parameters $M, N$ and the new parameter $q$. We can achieve up to $\frac{3}{2}$ speedup on the independent phase and up to $\frac{9}{5}$ speedup on the dependent phase. Since the dependent phase is expected to run $10^{2}-10^{4}$ times, the overall speedup is close to $\frac{9}{5}$.

We implemented the optimized algorithm and verified that runs with acceptable errors for small matrices. We also noted the numerical instability of the algorithm. Resolving this numerical stability is the direction of our research into this algorithm.

\section{References}

[1] S. K. Seal, K. P. Perumalla, and S. P. Hirshman, "Revisiting Parallel Cyclic Reduction and Parallel Prefix-based Algorithms for Block Tridiagonal Systems of Equations," Journal of Parallel and Distributed Computing, vol. 73, pp. 273-280, 2013.

[2] S. K. Seal, "An accelerated recursive doubling algorithm for block tridiagonal systems," in 2014 IEEE 28th International Parallel and Distributed Processing Symposium, 2014, pp. 1019-1028. 